\title{
A Straightforward Synthesis of Functionalized cis-Perhydroisoquinolin-1-ones
}

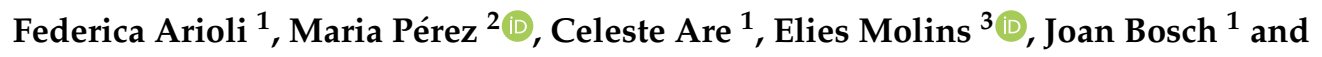 \\ Mercedes Amat 1,*D \\ 1 Laboratory of Organic Chemistry, Faculty of Pharmacy and Food Sciences, and Institute of \\ Biomedicine (IBUB), University of Barcelona, 08028 Barcelona, Spain; fede.arioli@hotmail.it (F.A.); \\ cele.are@ub.edu (C.A.); joanbosch@ub.edu (J.B.) \\ 2 Department of Nutrition, Food Sciences and Gastronomy, Faculty of Pharmacy and Food Sciences, \\ and Institute of Nutrition and Food Safety (INSA-UB), University of Barcelona, 08921 Santa Coloma de \\ Gramanet, Spain; mariaperez@ub.edu \\ 3 Institut de Ciència de Materials de Barcelona (ICMAB-CSIC), Campus UAB, 08193 Cerdanyola, Spain; \\ elies.molins@icmab.es \\ * Correspondence: amat@ub.edu; Tel.: +34-93-402-4540
}

Academic Editors: David Díez and María Ángeles Castro Received: 15 January 2019; Accepted: 30 January 2019; Published: 3 February 2019

\begin{abstract}
Base-catalyzed annulation reactions of 5,6-dihydro-2(1H)-pyridones with Nazarov-type reagents are reported. The effect of the solvent polarity and the concentration of the reagents is studied. The process involves two successive Michael additions and stereoselectively provides functionalized cis-perhydroisoquinolin-1-ones.
\end{abstract}

Keywords: 5,6-dihydro-2(1H)-pyridone; Nazarov reagents; perhydroisoquinoline; Michael addition; stereoselectivity

\section{Introduction}

Nitrogen heterocycles exhibit a broad range of significant biological and pharmacological activities, and many of them have been developed as therapeutic drugs [1,2].

In particular, the partially or totally reduced isoquinoline ring system is present in a large number of biologically active natural products (such as the alkaloids of the yohimbine [3,4], manzamine [5], and madangamine [6] groups) and medicinally useful synthetic compounds (e.g., the HIV protease inhibitors saquinavir and nelfinavir [7,8], the antimigraine drugs tezampanel and LY466195 [9], and the antiobesity agent AMG 076 [10]) (Figure 1).

Together with the intramolecular Diels-Alder cyclization of suitable azatrienes [11,12], one of the most straightforward approaches for the construction of the hydroisoquinoline ring system involves the generation of the carbocyclic ring by an annulation reaction from appropriate 5,6-dihydro-2(1H)-pyridone derivatives. The latter strategy was developed some years ago in our laboratory using the classical Diels-Alder methodology with a variety of dienes [13]. Bearing in mind that Nazarov reagents ( $\gamma, \delta$-unsaturated $\beta$-keto esters) are able to participate in double Michael addition reactions with $\alpha, \beta$-unsaturated carbonyl derivatives, we envisaged an alternative annulation procedure to directly access functionalized hydroisoquinolines from 5,6-dihydro-2(1H)-pyridones. 


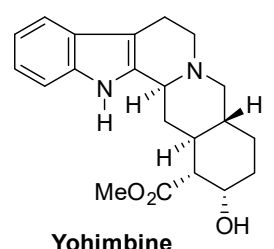

-BuHNOC_CH

Nelfinavir
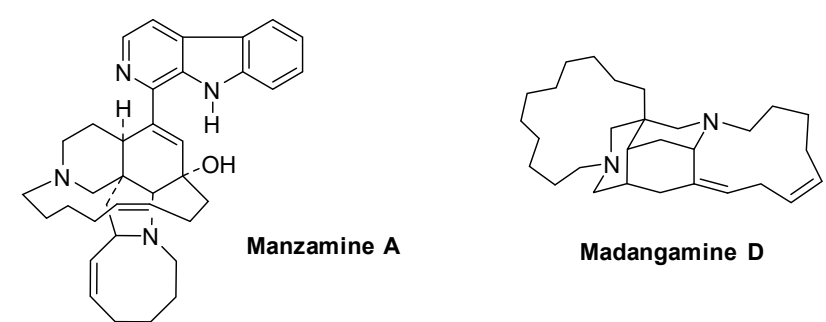

Madangamine D

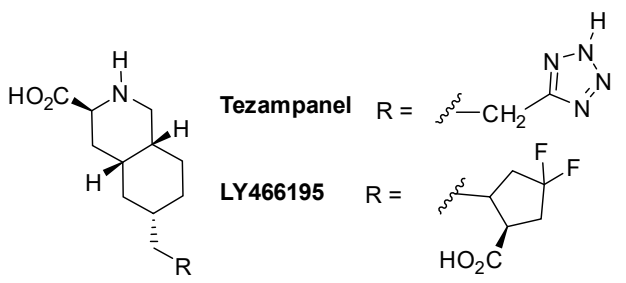

$\mathrm{HO}_{2} \mathrm{C}$

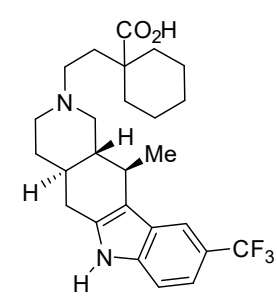

AMG 076

Figure 1. Hydroisoquinoline-containing bioactive natural products and synthetic compounds.

Nazarov reagents are versatile annulating agents, extensively used in a variety of Robinson-type and double Michael addition annulations [14-16]. In the former, the reagent sequentially acts as an electrophilic Michael acceptor and as a nucleophile to promote an aldol condensation. In the latter, however, it successively acts as a nucleophilic Michael donor and an electrophilic Michael acceptor, a reactivity pattern that has been successfully applied to assemble pentacyclic yohimbine-type derivatives from unsaturated indolo[2,3-a]quinolizidine-derived lactams [17-19].

\section{Results and Discussion}

Compounds $\mathbf{6 a}, \mathbf{b}, \mathbf{c}$ and $\mathbf{8}$, which incorporate an additional phenylsulfonyl or ethoxycarbonyl activating electron-withdrawing group conjugated to the carbon-carbon double bond, were selected as the starting dihydropyridones.

Lactams $\mathbf{6 a}, \mathbf{b}$, bearing an easily removable phenylsulfonyl group, were prepared in acceptable overall yield from 2-piperidone (1a) by bis-sulfenylation, followed by reaction with either (Boc) ${ }_{2} \mathrm{O}$ or MeI, and subsequent stepwise $m$-CPBA oxidation of the resulting $N$-substituted piperidones $\mathbf{3 a}, \mathbf{b}$ via unsaturated sulfenyl and sulfinyl derivatives $\mathbf{4 a}, \mathbf{b}$ and $\mathbf{5 a}, \mathbf{b}$. A similar reaction sequence from $\mathrm{N}$-tosyl-2-piperidone $3 \mathrm{c}$ led to unsaturated lactam $\mathbf{6 c}$ in low overall yield. Due to their instability, lactams $\mathbf{6 a , b}, \mathbf{c}$ were used in the annulation step without purification. In turn, ethoxycarbonyl lactam 8 was prepared in high yield from $N$-Boc-2-piperidone (1b) via seleno derivative 7 , as outlined in Scheme 1.

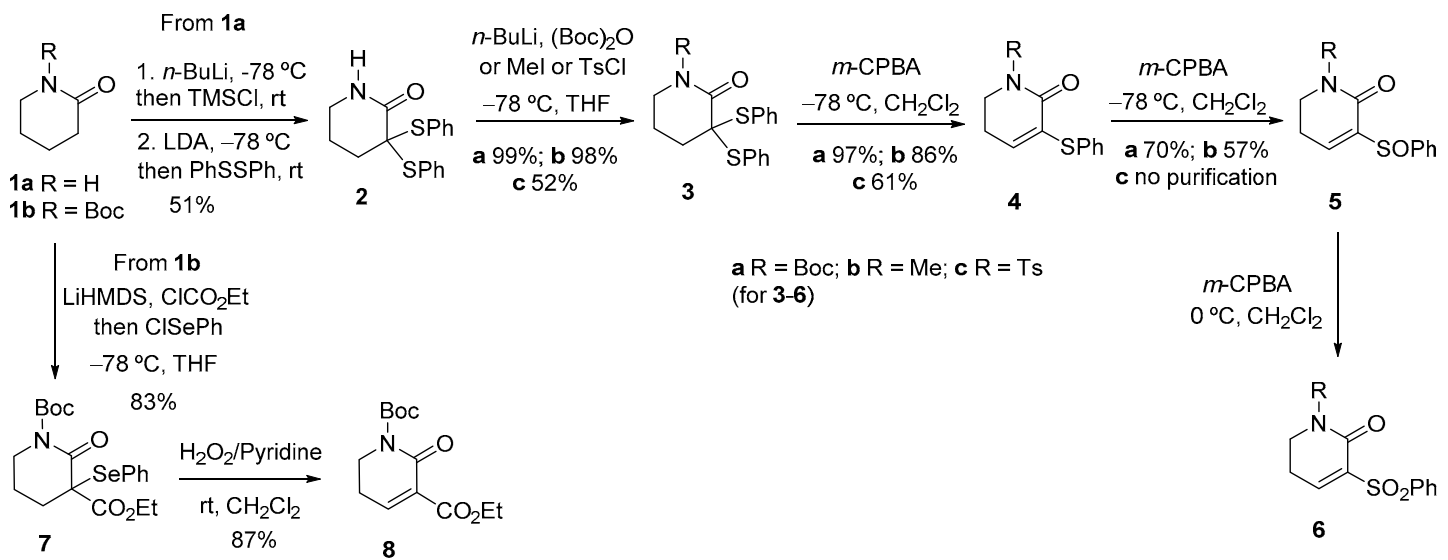

Scheme 1. Preparation of the starting unsaturated lactams. 
To study the annulation reactions, we initially used unsaturated lactam $6 \mathbf{a}$ and the Nazarov reagent 9 [14], which incorporates a methyl group at the terminal olefinic carbon, in the presence of $\mathrm{Cs}_{2} \mathrm{CO}_{3}$, the most commonly used base for the generation of Nazarov enolates [20]. Reagent 9 has been used extensively by Deslongchamps to generate cis-decalin derivatives [20,21]. The reaction was carried out at room temperature, using an excess (6 equiv.) of $\mathrm{Cs}_{2} \mathrm{CO}_{3}$ in $\mathrm{CH}_{2} \mathrm{Cl}_{2}$ at different concentrations (from $50 \mathrm{mM}$ to $5 \mathrm{mM}$ ). In all cases, the double Michael addition reaction occurred satisfactorily, although with only moderate stereoselectivity, to give 3:1 C-8 stereoisomeric mixtures of cis-hydroisoquinolones $10 \mathbf{a}$ (cis $\mathrm{Me} / \mathrm{SO}_{2} \mathrm{Ph}$ ) and $\mathbf{1 0 b}$ (trans $\mathrm{Me} / \mathrm{SO}_{2} \mathrm{Ph}$ ), the chemical yield increasing (79\% yield at $5 \mathrm{mM}$ ) with the dilution (Table 1, entries 1-3). Trace amounts of the monoaddition product 11 were detected by NMR, indicating that the annulation involves two successive Michael addition reactions. In contrast, when acetonitrile was used as the solvent the yield was very low (entry 4). Remarkably, the use of KF as the base in a polar solvent such as methanol (entry 5) resulted in a reversal of the stereoselectivity, leading to a mixture of bicyclic lactams $10 \mathbf{a}$ and $10 \mathbf{b}$, in which the trans $\mathrm{Me} / \mathrm{SO}_{2} \mathrm{Ph}$ isomer predominated (56\% yield, ratio 1:2). The influence of the solvent polarity on the stereoselectivity of annulation reactions of 9 with $\beta$-keto esters has previously been observed [21]. The facial selectivity when using $\mathrm{Cs}_{2} \mathrm{CO}_{3}$ as the base can be attributed to the coordination of the $\mathrm{Cs}^{+}$ cation to the oxygen atoms of both the Nazarov reagent and the starting lactam [19].

Table 1. Reaction of unsaturated lactam 6 a with Nazarov reagent 9.

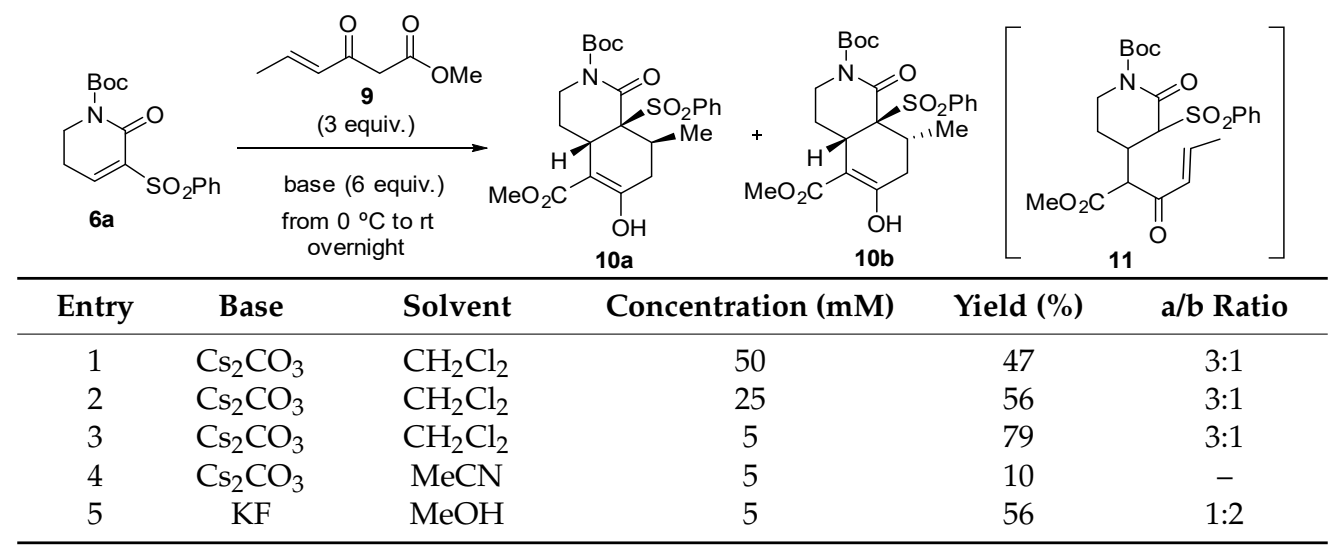

The relative $\mathrm{Me} / \mathrm{SO}_{2} \mathrm{Ph}$ cis configuration of bicyclic lactam 10a was unambiguously established by X-ray crystallographic analysis (Figure 2).

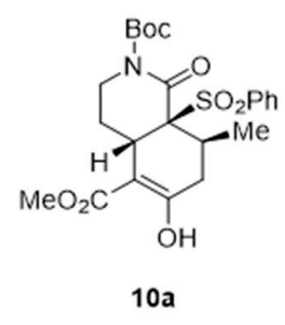

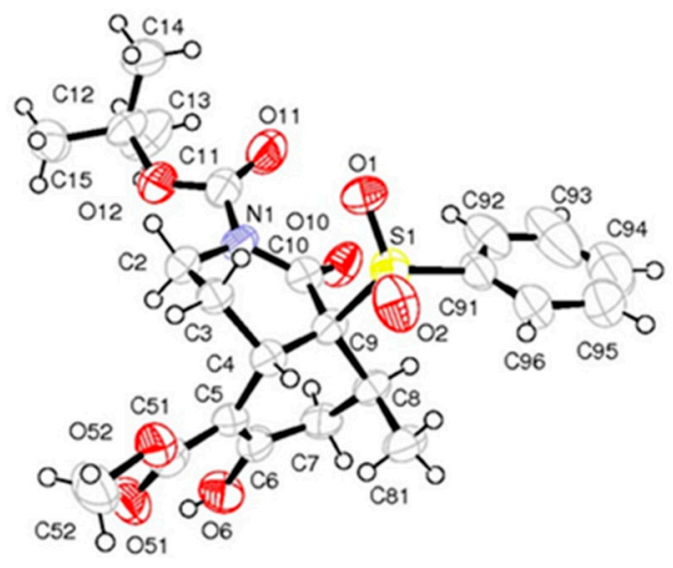

Figure 2. ORTEP plot of the X-ray structure of bicyclic lactam 10a.

Unsaturated lactam $\mathbf{6 b}$ behaved similarly to lactam $\mathbf{6} \mathbf{a}$ in the annulation reaction with Nazarov reagent 9 , although the yields were slightly lower, probably due to the lower electrophilicity of the 
Michael acceptor as a consequence of the absence of an electron-withdrawing group on the piperidone nitrogen. cis-Hydroisoquinolone $\mathbf{1 2 a}$, with a cis $\mathrm{Me} / \mathrm{SO}_{2} \mathrm{Ph}$ relationship, was stereo-selectively formed (4:1 12a/12b ratio) when the reaction was performed in $\mathrm{CH}_{2} \mathrm{Cl}_{2}$ solution using $\mathrm{Cs}_{2} \mathrm{CO}_{3}$ as the base (Table 2, entries 1 and 2), the yield once again being higher with increasing dilution (40\% yield at $5 \mathrm{mM}$ ). As before, a reversal of the stereoselectivity was observed and the trans $\mathrm{Me} / \mathrm{SO}_{2} \mathrm{Ph}$ isomer predominated when using polar solvents, either $\mathrm{MeOH}$ in the presence of KF (1:4 ratio; entry 3) or DMF in the presence of $\mathrm{Cs}_{2} \mathrm{CO}_{3}$ (1:5 ratio; entry 4). The $\mathrm{Me} / \mathrm{SO}_{2} \mathrm{Ph}$ cis relationship of the adducts 10a and 12a was maintained unchanged after an additional treatment $(20 \mathrm{~h}, \mathrm{rt})$ with $\mathrm{Cs}_{2} \mathrm{CO}_{3}$ in $\mathrm{CH}_{2} \mathrm{Cl}_{2}$ or $\mathrm{KF}$ in $\mathrm{MeOH}$, thus suggesting the non-reversibility of the cyclization step.

Table 2. Reaction of unsaturated lactam $6 b$ with Nazarov reagent 9.

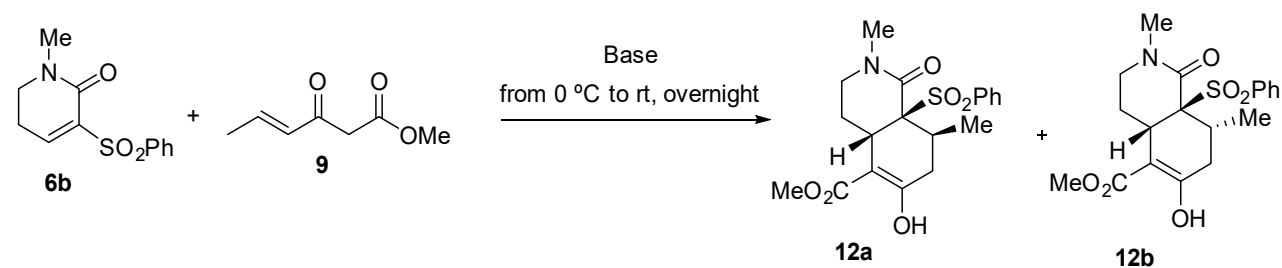

\begin{tabular}{cccccccc}
\hline Entry & Base & Equiv Base & Equiv (9) & Solvent & Concentration (mM) & Yield (\%) & a/b Ratio \\
\hline 1 & $\mathrm{Cs}_{2} \mathrm{CO}_{3}$ & 6 & 3 & $\mathrm{CH}_{2} \mathrm{Cl}_{2}$ & 100 & 28 & $4: 1$ \\
2 & $\mathrm{Cs}_{2} \mathrm{CO}_{3}$ & 6 & 3 & $\mathrm{CH}_{2} \mathrm{Cl}_{2}$ & 5 & 40 & $4: 1$ \\
3 & $\mathrm{KF}$ & 6 & 3 & $\mathrm{MeOH}$ & 5 & 51 & $1: 4$ \\
4 & $\mathrm{Cs}_{2} \mathrm{CO}_{3}$ & 4 & 2 & $\mathrm{DMF}$ & 5 & 45 & $1: 5$ \\
\hline
\end{tabular}

The relative configuration of both hydroisoquinolones, $\mathbf{1 2 a}$ and $\mathbf{1 2} \mathbf{b}$, was unambiguously established by X-ray crystallographic analysis (Figure 3).
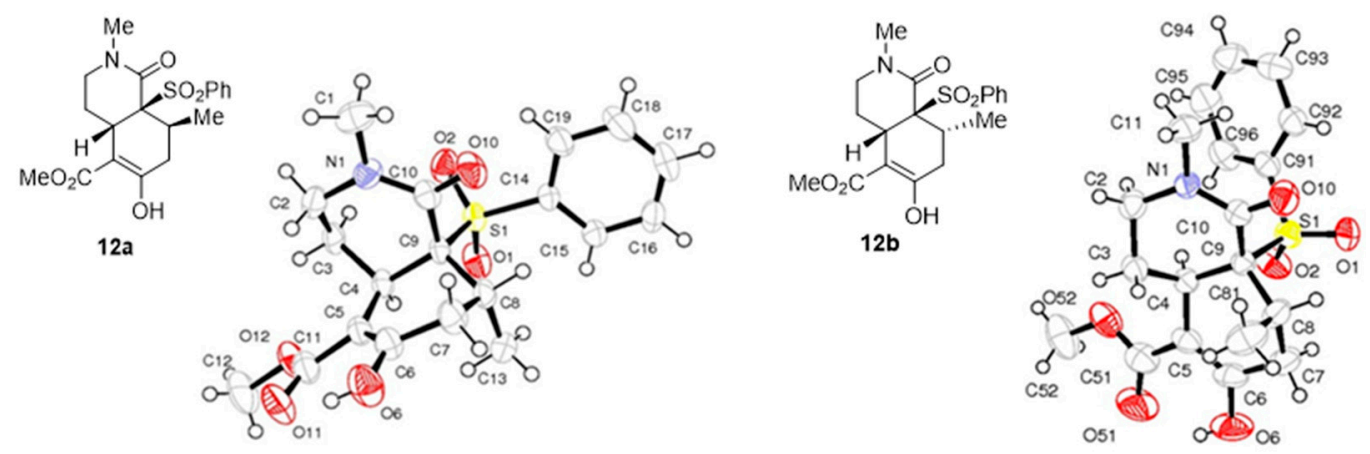

Figure 3. ORTEP plots of the X-ray structures of bicyclic lactams 12a and 12b.

The activating phenylsulfonyl group of bicyclic lactams 10a, 12a, and $\mathbf{1 2 b}$ was stereoselectively removed, with retention of configuration, by treatment with sodium amalgam [22] to give the respective cis-hydroisoquinolones 13a, 14a, and 14b (Scheme 2). Alternatively, removal of the N-Boc protecting group of hydroisoquionolones 10a and 13a quantitatively afforded the potentially useful $N$-unsubstituted derivatives 15 and 16, respectively. The cis ring function was evident from the observation of a positive NOE effect between the 4a and 8a methine protons.

To expand the scope of the methodology and access hydroisoquinolones lacking the methyl substituent on the B ring, we decided to study double Michael annulations using the silylated Nazarov reagent 17 [17], which constitutes a stable synthetic equivalent of the original Nazarov reagent (methyl or ethyl 3-oxo-4-pentenoate) [15] that avoids the polymerization problems associated with the latter under basic conditions. 


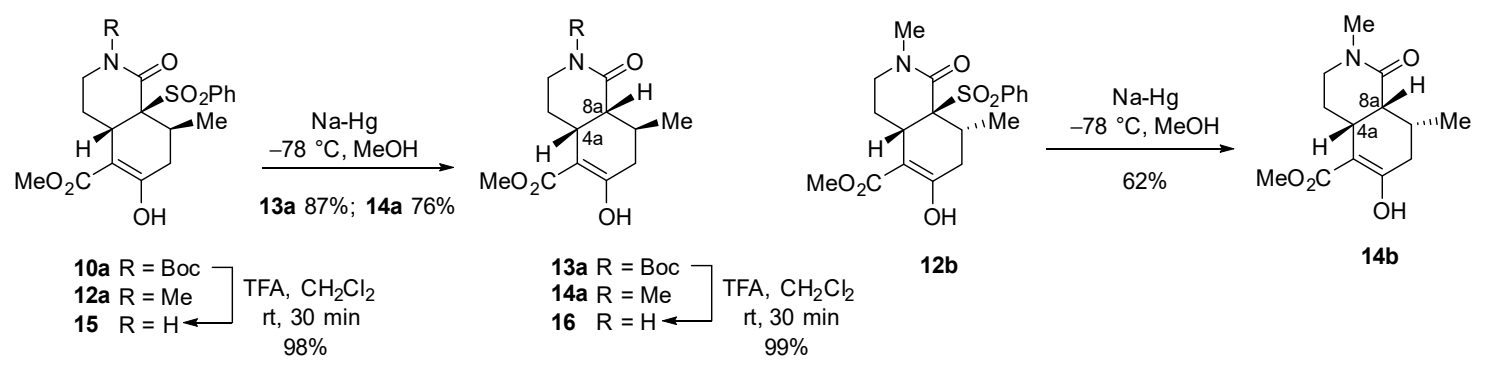

Scheme 2. Desulfonylation reactions and removal of the N-Boc protecting group.

As expected, the $\mathrm{Cs}_{2} \mathrm{CO}_{3}$-promoted annulation of the Nazarov reagent 17 with unsaturated lactam 6a under the usual reaction conditions ( $5 \mathrm{mM}$ in $\mathrm{CH}_{2} \mathrm{Cl}_{2}$ as the solvent) stereoselectively afforded cis-hydroisoquinolone 18a, in which protodesilylation had occurred, in acceptable yield (Table 3, entry 1). The yield was not improved by increasing the excess of reagent and base (entry 2) and was lower when operating at a higher concentration (entry 3) or when using $\mathrm{KF}$ in $\mathrm{MeOH}$ as the solvent (entry 4). Similar moderate yields were obtained in the generation of cis-hydroisoquinolones $\mathbf{1 8 b}$ and $\mathbf{1 8 c}$ from lactams $\mathbf{6 b}$ (entries 5-7) and $\mathbf{6 c}$ (entry 8 ) under a variety of conditions.

Table 3. Reactions of unsaturated lactams 6a-c with Nazarov reagent 17.

\begin{tabular}{|c|c|c|c|c|c|c|c|c|}
\hline & $\begin{array}{l}R \\
I\end{array}$ & & 17 & $\frac{\mathrm{fr}}{\mathrm{a} R}$ & $\begin{array}{c}\text { Base } \\
0^{\circ} \mathrm{C} \text { to rt, o } \\
\text { Boc; } b \mathrm{R}=\mathrm{M}\end{array}$ & $\underset{c R=T s}{\stackrel{\text { rnight }}{\longrightarrow}}$ & $Y O$ & \\
\hline Entry & $\begin{array}{l}\text { Unsaturated } \\
\text { Lactam }\end{array}$ & Base & $\begin{array}{l}\text { Equiv } \\
\text { Base }\end{array}$ & $\begin{array}{l}\text { Equiv } \\
\text { (15) }\end{array}$ & Solvent & $\begin{array}{c}\text { Concentration } \\
(\mathrm{mM})\end{array}$ & Product & Yield (\%) \\
\hline 1 & $6 a$ & $\mathrm{Cs}_{2} \mathrm{CO}_{3}$ & 6 & 3 & $\mathrm{CH}_{2} \mathrm{Cl}_{2}$ & 5 & $18 a$ & 47 \\
\hline 2 & $6 a$ & $\mathrm{Cs}_{2} \mathrm{CO}_{3}$ & 10 & 5 & $\mathrm{CH}_{2} \mathrm{Cl}_{2}$ & 5 & $18 a$ & 47 \\
\hline 3 & $6 a$ & $\mathrm{Cs}_{2} \mathrm{CO}_{3}$ & 6 & 3 & $\mathrm{CH}_{2} \mathrm{Cl}_{2}$ & 20 & $18 a$ & 20 \\
\hline 4 & $6 a$ & KF & 6 & 3 & $\mathrm{MeOH}$ & 5 & $18 a$ & 20 \\
\hline 5 & $6 b$ & $\mathrm{Cs}_{2} \mathrm{CO}_{3}$ & 6 & 3 & $\mathrm{CH}_{2} \mathrm{Cl}_{2}$ & 5 & $18 b$ & 35 \\
\hline 6 & $6 b$ & $\mathrm{Cs}_{2} \mathrm{CO}_{3}$ & 6 & 3 & $\mathrm{CH}_{2} \mathrm{Cl}_{2}$ & 100 & $18 b$ & 21 \\
\hline 7 & $6 b$ & KF & 6 & 3 & $\mathrm{MeOH}$ & 5 & $18 b$ & 20 \\
\hline 8 & $6 c$ & $\mathrm{Cs}_{2} \mathrm{CO}_{3}$ & 6 & 3 & $\mathrm{CH}_{2} \mathrm{Cl}_{2}$ & 5 & $18 \mathrm{c}$ & 32 \\
\hline
\end{tabular}

Remarkably, the yield of the annulation with the silylated Navarov reagent 17 was higher when using unsaturated lactam 8, which incorporates an ester group as an additional activating substituent. Operating under the previously optimized reaction conditions, cis-hydroisoquinolone 19 was obtained in $60 \%$ yield (Scheme 3 ).

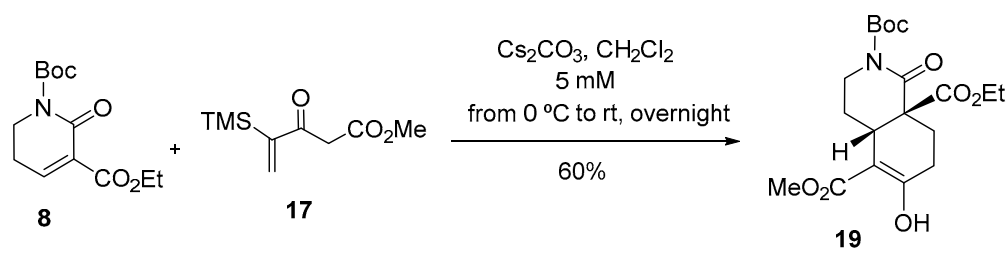

Scheme 3. Reaction of unsaturated lactam 8 with the silylated Nazarov reagent 17.

In conclusion, base-promoted annulation reactions of Nazarov reagents 9 and $\mathbf{1 7}$ with 5,6-dihydro-2 $(1 H)$-pyridones bearing an additional activating electron-withdrawing group $\alpha$ to the lactam carbonyl constitute a straightforward procedure for the stereoselective synthesis of highly substituted cis-hydroisoquinolin-2-ones. In the reactions with the methyl-substituted reagent 9 , leading 
to 8-substituted derivatives, the use of $\mathrm{Cs}_{2} \mathrm{CO}_{3}$ in $\mathrm{CH}_{2} \mathrm{Cl}_{2}$ leads to cis-hydroisoquinolones with a cis $8-\mathrm{Me} / 8 \mathrm{a}-\mathrm{SO}_{2} \mathrm{Ph}$ relationship as the major stereoisomers. The stereoselectivity is reversed in a polar solvent such as DMF or when the annulation is performed using KF in $\mathrm{MeOH}$.

The methodology developed here provides access to polyfunctionalized bicyclic scaffolds with potential use as precursors of bioactive hydroisoquinoline-containing natural products and synthetic derivatives.

\section{Materials and Methods}

\subsection{General Information}

All air sensitive manipulations were carried out under a dry argon or nitrogen atmosphere. THF and $\mathrm{CH}_{2} \mathrm{Cl}_{2}$ were dried using a column solvent purification system. Analytical thin-layer chromatography was performed on $\mathrm{SiO}_{2}$ (silica gel 60A 35-70 $\mu \mathrm{m}$, Carlo Erba, Val de Reuil Cedex, France), and the spots were located with $1 \%$ aqueous $\mathrm{KMnO}_{4}$. Chromatography refers to flash chromatography and was carried out on $\mathrm{SiO}_{2}$ (SDS silica gel 60 ACC, 35-75 mm, 230-240 mesh ASTM). NMR spectra were recorded at 300 or $400 \mathrm{MHz}\left({ }^{1} \mathrm{H}\right)$ and $100.6 \mathrm{MHz}\left({ }^{13} \mathrm{C}\right)$, and chemical shifts are reported in $\delta$ values downfield from TMS or relative to residual chloroform $(7.26 \mathrm{ppm}$, $77.0 \mathrm{ppm}$ ) as an internal standard. Data are reported in the following manner: chemical shift, multiplicity, coupling constant $(\mathrm{J})$ in hertz $(\mathrm{Hz})$, integrated intensity, and assignment (when possible). Assignments and stereochemical determinations are given only when they are derived from definitive two-dimensional NMR experiments (HSQC-COSY). IR spectra were performed in an Avatar 320 FT-IR spectrophotometer (Thermo Nicolet, Madison, WI, USA) and only noteworthy IR absorptions $\left(\mathrm{cm}^{-1}\right)$ are listed. High resolution mass spectra (HMRS; LC/MSD TOF, Agilent Technologies, Santa Clara, CA, USA) were performed by Centres Científics i Tecnològics de la Universitat de Barcelona.

\subsection{Preparation of the Starting Unsaturated Lactams}

3,3-Bis(phenylthio)-2-piperidone (2). $n$-BuLi (25.5 $\mathrm{mL}$ of a $1.6 \mathrm{M}$ solution in hexane, $40.8 \mathrm{mmol}$ ) was added under an argon atmosphere at $-78{ }^{\circ} \mathrm{C}$ to a solution of $\delta$-valerolactam (1a, $\left.4 \mathrm{~g}, 40.82 \mathrm{mmol}\right)$ in anhydrous THF (100 mL). The solution was stirred for 30 minutes, and then TMSCl (5.2 mL, $40.8 \mathrm{mmol})$ was added. The solution was stirred at room temperature for 30 minutes, and then re-cooled to $-78{ }^{\circ} \mathrm{C}$. LDA ( $45 \mathrm{~mL}, 2 \mathrm{M}$ solution in THF/heptane/ethylbenzene, $89.8 \mathrm{mmol}$ ) was added, and the solution was stirred for 30 minutes. Then, PhSSPh $(19.5 \mathrm{~g}, 89.8 \mathrm{mmol})$ in anhydrous THF $(50 \mathrm{~mL})$ was added, and the resulting solution was stirred at room temperature for $1 \mathrm{~h}$. After this time, $\mathrm{NH}_{4} \mathrm{Cl}$ was added, and the organic layer was extracted with EtOAc. The combined organic extracts were dried over anhydrous $\mathrm{MgSO}_{4}$, filtered, and concentrated under reduced pressure. Flash chromatography (9:1 hexane-EtOAc) of the residue gave lactam $2\left(6.61 \mathrm{~g}, 51 \%\right.$ yield): IR (ATR Pike) $v\left(\mathrm{~cm}^{-1}\right): 3260$ $(\mathrm{NH}), 1661(\mathrm{CO}) ;{ }^{1} \mathrm{H}-\mathrm{NMR}\left(400 \mathrm{MHz}, \mathrm{CDCl}_{3}\right): \delta=1.72-1.90(\mathrm{~m}, 2 \mathrm{H}, \mathrm{H}-5), 1.97(\mathrm{t}, J=6.2 \mathrm{~Hz}, 2 \mathrm{H}$, $\mathrm{H}-4), 3.18(\mathrm{dt}, J=2.4,6.0 \mathrm{~Hz}, 2 \mathrm{H}, \mathrm{H}-6), 6.26$ (br. s, $1 \mathrm{H}, \mathrm{NH}), 7.32-7.34\left(\mathrm{~m}, 6 \mathrm{H}, \mathrm{H}_{\mathrm{AR}}\right), 7.63-7.65(\mathrm{~m}$, $4 \mathrm{H}, \mathrm{H}_{\mathrm{AR}}$ ); HRMS (ESI) calcd for $\left[\mathrm{C}_{17} \mathrm{H}_{18} \mathrm{NOS}_{2}+\mathrm{H}^{+}\right]$: 316.0824 , found: $316.062714 \mathrm{~b}$ (detailed data in supplementary materials).

3,3-Bis(phenylthio)-1-(tert-butoxycarbonyl)-2-piperidone (3a). $n$-BuLi (6.1 $\mathrm{mL}$ of a $1.4 \mathrm{M}$ solution in hexane, $8.54 \mathrm{mmol})$ was added under an argon atmosphere at $-78{ }^{\circ} \mathrm{C}$ to a solution of lactam $2(2.25 \mathrm{~g}$, $7.12 \mathrm{mmol})$ in anhydrous THF $(100 \mathrm{~mL})$, and the resulting solution was stirred for $1 \mathrm{~h}$. Then, $\mathrm{Boc}_{2} \mathrm{O}$ $(2.30 \mathrm{~g}, 10.68 \mathrm{mmol})$ was added, and the mixture was stirred at $-78{ }^{\circ} \mathrm{C}$ for $1 \mathrm{~h}$. After this time, saturated $\mathrm{NH}_{4} \mathrm{Cl}$ was added, and the mixture was extracted with EtOAc. The combined organic extracts were dried over $\mathrm{Na}_{2} \mathrm{SO}_{4}$ and filtered, and the solvent was removed under reduced pressure. Flash chromatography (9:1 hexane-EtOAc) of the residue gave $N$-Boc derivative $3 a$ ( $2.92 \mathrm{~g}, 99 \%$ yield): IR (ATR Pike) $v\left(\mathrm{~cm}^{-1}\right): 1717(\mathrm{CO}) ;{ }^{1} \mathrm{H}-\mathrm{NMR}\left(400 \mathrm{MHz}, \mathrm{CDCl}_{3}\right): \delta=1.53\left[\mathrm{~s}, 9 \mathrm{H},\left(\mathrm{CH}_{3}\right)_{3} \mathrm{C}\right], 1.94(\mathrm{~m}$, 2H, H-5), 2.05 (m, 2H, H-4), $3.60(\mathrm{t}, J=6.1 \mathrm{~Hz}, 2 \mathrm{H}, \mathrm{H}-6), 7.33-7.42\left(\mathrm{~m}, 6 \mathrm{H}, \mathrm{H}_{\mathrm{AR}}\right), 7.68\left(\mathrm{~m}, 4 \mathrm{H}, \mathrm{H}_{\mathrm{AR}}\right)$; ${ }^{13} \mathrm{C}-\mathrm{NMR}\left(400 \mathrm{MHz}, \mathrm{CDCl}_{3}\right): \delta=20.1(\mathrm{C}-5), 28.0\left[\left(\mathrm{CH}_{3}\right)_{3} \mathrm{C}\right], 35.1$ (C-4), 46.7 (C-6), 68.6 (C-3), 83.2 
$\left[\left(\mathrm{CH}_{3}\right)_{3} \mathrm{C}\right], 128.7\left(4 \mathrm{CH}_{\mathrm{AR}}\right), 129.6\left(2 \mathrm{CH}_{\mathrm{AR}}\right), 130.9\left(2 \mathrm{C}_{\mathrm{AR}}\right), 136.8\left(4 \mathrm{CH}_{\mathrm{AR}}\right), 153.8(\mathrm{CO}), 168.3(\mathrm{CO}) ; \mathrm{HRMS}$ (ESI) calcd for $\left[\mathrm{C}_{22} \mathrm{H}_{25} \mathrm{NO}_{3} \mathrm{~S}_{2}+\mathrm{Na}^{+}\right]$: 438.1168, found: 438.117 .

3,3-Bis(phenylthio)-1-methyl-2-piperidone (3b). $n$-BuLi (8.54 $\mathrm{mL}$ of a 1.6 M solution in hexane, $13.67 \mathrm{mmol})$ was added under an argon atmosphere at $-78{ }^{\circ} \mathrm{C}$ to a solution of lactam $2(3.60 \mathrm{~g}, 11.39 \mathrm{mmol}) \mathrm{in}$ anhydrous THF $(200 \mathrm{~mL})$, and the resulting mixture was stirred at $-78^{\circ} \mathrm{C}$ for $1 \mathrm{~h}$. Then, MeI $(1.06 \mathrm{~mL}$, $17.09 \mathrm{mmol}$ ) was added, and the solution was stirred at room temperature for $1 \mathrm{~h}$. The reaction was quenched by addition of saturated $\mathrm{NH}_{4} \mathrm{Cl}$, and the mixture was extracted with EtOAc. The combined organic extracts were dried over $\mathrm{Na}_{2} \mathrm{SO}_{4}$, filtered, and concentrated under reduced pressure. Flash chromatography (9:1 hexane-EtOAc) of the residue afforded $N$-methyl derivative $3 \mathrm{~b}(3.7 \mathrm{~g}, 98 \%$ yield): IR (ATR Pike) v (cm $\left.{ }^{-1}\right): 1642(\mathrm{NCO}) ;{ }^{1} \mathrm{H}-\mathrm{NMR}\left(400 \mathrm{MHz}, \mathrm{CDCl}_{3}\right): \delta=1.86-1.91(\mathrm{~m}, 2 \mathrm{H}, \mathrm{H}-5), 1.97-1.99$ (m, 2H, H-4), $2.92\left(\mathrm{~s}, 3 \mathrm{H}, \mathrm{CH}_{3}\right), 3.16(\mathrm{t}, J=6.4 \mathrm{~Hz}, 2 \mathrm{H}, \mathrm{H}-6), 7.31-7.38\left(\mathrm{~m}, 6 \mathrm{H}, \mathrm{H}_{\mathrm{AR}}\right), 7.63-7.65(\mathrm{~m}$, $\left.4 \mathrm{H}, \mathrm{H}_{\mathrm{AR}}\right) ;{ }^{13} \mathrm{C}-\mathrm{NMR}\left(100.6 \mathrm{MHz} \mathrm{CDCl}_{3}\right): \delta=19.6(\mathrm{C}-5), 34.0(\mathrm{C}-4), 35.8\left(\mathrm{CH}_{3}\right), 50.1(\mathrm{C}-6), 66.0(\mathrm{C}-3)$, $128.6\left(4 \mathrm{CH}_{\mathrm{AR}}\right), 129.3\left(2 \mathrm{CH}_{\mathrm{AR}}\right), 131.6\left(2 \mathrm{C}_{\mathrm{AR}}\right), 136.6\left(4 \mathrm{CH}_{\mathrm{AR}}\right) ; \mathrm{HRMS}(\mathrm{ESI})$ calcd for $\left[\mathrm{C}_{18} \mathrm{H}_{19} \mathrm{NOS}_{2}+\mathrm{H}^{+}\right]$: 330.0981, found: 330.0977 .

3,3-Bis(phenylthio)-1-(p-toluenesulfonyl)-2-piperidone (3c). $n$-BuLi (1.2 mL of a 1.6 M solution in hexane, $1.9 \mathrm{mmol})$ was added at $-78^{\circ} \mathrm{C}$ to a solution of lactam $2(500 \mathrm{mg}, 1.58 \mathrm{mmol})$ in anhydrous THF $(25 \mathrm{~mL})$, and the resulting mixture was stirred at $-78{ }^{\circ} \mathrm{C}$ for $1 \mathrm{~h}$. Then, $\mathrm{TsCl}(452 \mathrm{mg}, 2.37 \mathrm{mmol})$ was added, and the solution was stirred at room temperature overnight. The reaction was quenched by addition of saturated $\mathrm{NH}_{4} \mathrm{Cl}$, and the mixture was extracted with EtOAc. The combined organic extracts were dried over $\mathrm{Na}_{2} \mathrm{SO}_{4}$, filtered, and concentrated under reduced pressure. Flash chromatography (9:1 hexane-EtOAc) afforded $N$-tosyl derivative 3c (264 mg, 52\% yield): IR (ATR Pike) $v\left(\mathrm{~cm}^{-1}\right): 1683$ (NCO), $1169\left(\mathrm{SO}_{2}\right) ;{ }^{1} \mathrm{H}-\mathrm{NMR}\left(400 \mathrm{MHz}, \mathrm{CDCl}_{3}\right): \delta=1.55$ (s, 2H, H-5), 2.02 (s, 2H, H-4), 2.49 (s, 3H, $\mathrm{CH}_{3}$ ), 3.46 (br. s, 2H, H-6), 7.19-7.40 (m, 12H, $\mathrm{H}_{\mathrm{AR}}$ ), 7.93 (d, J = 8.4 Hz, 2H, $\mathrm{H}_{\mathrm{AR}}$ ).

1-(tert-Butoxycarbonyl)-3-(phenylthio)-5,6-dihydro-2(1H)-pyridone (4a). m-Chloroperbenzoic acid (1.09 g, technical grade $70 \%, 766 \mathrm{mg}$ pure oxidant, $6.33 \mathrm{mmol})$ in $\mathrm{CH}_{2} \mathrm{Cl}_{2}(100 \mathrm{~mL})$ was added to a solution of lactam $3 \mathrm{a}(2.64 \mathrm{~g}, 6.33 \mathrm{mmol})$ in $\mathrm{CH}_{2} \mathrm{Cl}_{2}(200 \mathrm{~mL})$ at $-78{ }^{\circ} \mathrm{C}$. The solution was stirred at this temperature for $2 \mathrm{~h}$ and then for 30 minutes at room temperature. After this time, a saturated solution of $\mathrm{NaHCO}_{3}$ was added, the mixture was extracted with $\mathrm{CH}_{2} \mathrm{Cl}_{2}$, and the organic solvent was removed under reduced pressure. The crude product was purified by flash chromatography (95:5 hexane-EtOAc) to afford unsaturated lactam $4 \mathrm{a}\left(1.88 \mathrm{~g}, 97 \%\right.$ yield): IR (ATR Pike) $v\left(\mathrm{~cm}^{-1}\right)$ : 1762 (CO), 1715 (CO); ${ }^{1} \mathrm{H}-\mathrm{NMR}\left(300 \mathrm{MHz}, \mathrm{CDCl}_{3}\right): \delta=1.54\left[\mathrm{~s}, 9 \mathrm{H},\left(\mathrm{CH}_{3}\right)_{3} \mathrm{C}\right], 2.39(\mathrm{td}, J=6.6,4.8 \mathrm{~Hz}, 2 \mathrm{H}, \mathrm{H}-5), 3.84(\mathrm{t}$, $J=6.6 \mathrm{~Hz}, 2 \mathrm{H}, \mathrm{H}-6), 6.08(\mathrm{t}, J=4.8 \mathrm{~Hz}, 1 \mathrm{H}, \mathrm{H}-4), 7.33-7.41\left(\mathrm{~m}, 2 \mathrm{H}, \mathrm{H}_{\mathrm{AR}}\right), 7.47-7.50\left(\mathrm{~m}, 2 \mathrm{H}, \mathrm{H}_{\mathrm{AR}}\right)$, 7.66-7.70 (m, 1H, H $\mathrm{AR}) ;{ }^{13} \mathrm{C}-\mathrm{NMR}\left(100.6 \mathrm{MHz} \mathrm{CDCl}_{3}\right): \delta=25.3(\mathrm{C}-5), 28.0\left[\left(\mathrm{CH}_{3}\right)_{3} \mathrm{C}\right], 43.7(\mathrm{C}-6), 83.4$ $\left[\left(\mathrm{CH}_{3}\right)_{3} \mathrm{C}\right], 128.8\left(\mathrm{CH}_{\mathrm{AR}}\right), 129.5\left(2 \mathrm{CH}_{\mathrm{AR}}\right), 131.0\left(\mathrm{C}-3, \mathrm{C}_{\mathrm{AR}}\right), 134.8\left(2 \mathrm{CH}_{\mathrm{AR}}\right), 135.5(\mathrm{C}-4), 152.8(\mathrm{CO}), 161.7$ (CO); HRMS (ESI) calcd for $\left[\mathrm{C}_{16} \mathrm{H}_{19} \mathrm{NO}_{3} \mathrm{~S}+\mathrm{Na}^{+}\right]$: 328.0978, found: 328.0975 .

1-Methyl-3-(phenylthio)-5,6-dihydro-2(1H)-pyridone (4b). Operating as described for the preparation of compound $4 \mathbf{a}$, from a solution of lactam $3 \mathbf{b}(280 \mathrm{mg}, 0.85 \mathrm{mmol})$ in $\mathrm{CH}_{2} \mathrm{Cl}_{2}(20 \mathrm{~mL})$ and $m$-CPBA (210 mg, technical grade $70 \%, 0.85 \mathrm{mmol})$ in $\mathrm{CH}_{2} \mathrm{Cl}_{2}(20 \mathrm{~mL})$, unsaturated lactam $4 \mathbf{b}$ was obtained (160 mg, 86\% yield) as a yellow foam: ${ }^{1} \mathrm{H}-\mathrm{NMR}\left(400 \mathrm{MHz}, \mathrm{CDCl}_{3}, \mathrm{COSY}, \mathrm{g}-\mathrm{HSQC}\right): \delta=2.34(\mathrm{td}, J=7.2$, $4.8 \mathrm{~Hz}, 2 \mathrm{H}, \mathrm{H}-5), 3.04\left(\mathrm{~s}, 1 \mathrm{H}, \mathrm{CH}_{3}\right), 3.40(\mathrm{t}, J=7.2 \mathrm{~Hz}, 2 \mathrm{H}, \mathrm{H}-6), 5.81(\mathrm{t}, J=4.8 \mathrm{~Hz}, 1 \mathrm{H}, \mathrm{H}-4), 7.35-7.39$ $\left(\mathrm{m}, 3 \mathrm{H}, \mathrm{H}_{\mathrm{AR}}\right), 7.48-7.50\left(\mathrm{~m}, 2 \mathrm{H}, \mathrm{H}_{\mathrm{AR}}\right) ;{ }^{13} \mathrm{C}-\mathrm{NMR}\left(100.6 \mathrm{MHz}, \mathrm{CDCl}_{3}\right): \delta=24.6(\mathrm{C}-5), 34.9\left(\mathrm{CH}_{3}\right), 47.6$ $(\mathrm{C}-6), 128.5\left(\mathrm{CH}_{\mathrm{AR}}\right), 129.4\left(2 \mathrm{CH}_{\mathrm{AR}}\right), 130.8(\mathrm{C}-4), 132.0,134.4\left(\mathrm{C}-3, \mathrm{C}_{\mathrm{AR}}\right), 134.6\left(2 \mathrm{CH}_{\mathrm{AR}}\right), 162.9(\mathrm{CO})$.

3-(Phenylthio)-1-(p-toluenesulfonyl)-5,6-dihydro-2(1H)-pyridone (4c). Operating as described for the preparation of compound $4 \mathbf{a}$, from a solution of lactam $3 \mathbf{c}(264 \mathrm{mg}, 0.58 \mathrm{mmol})$ in $\mathrm{CH}_{2} \mathrm{Cl}_{2}(15 \mathrm{~mL})$ and $m$-CPBA (142 mg, technical grade $70 \%, 0.58 \mathrm{mmol})$ in $\mathrm{CH}_{2} \mathrm{Cl}_{2}(15 \mathrm{~mL})$, unsaturated lactam $4 \mathrm{c}$ was obtained (123 mg, 61\% yield) as a yellow foam: IR (ATR Pike) $v\left(\mathrm{~cm}^{-1}\right): 1683(\mathrm{CO}), 1166\left(\mathrm{SO}_{2}\right)$;

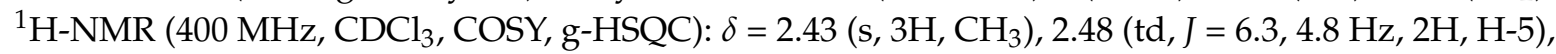
$4.04(\mathrm{t}, J=6.3 \mathrm{~Hz}, 2 \mathrm{H}, \mathrm{H}-6), 6.08(\mathrm{t}, J=4.8 \mathrm{~Hz}, 1 \mathrm{H}, \mathrm{H}-4), 7.30-7.37\left(\mathrm{~m}, 7 \mathrm{H}, \mathrm{H}_{\mathrm{AR}}\right), 7.93(\mathrm{~d}, J=8.4 \mathrm{~Hz}$, 
2H, $\left.\mathrm{H}_{\mathrm{AR}}\right) ;{ }^{13} \mathrm{C}-\mathrm{NMR}\left(100.6 \mathrm{MHz}, \mathrm{CDCl}_{3}\right): \delta=21.6\left(\mathrm{CH}_{3}\right), 25.9(\mathrm{C}-5), 44.1(\mathrm{C}-6), 128.7\left(\mathrm{CH}_{\mathrm{AR}}\right), 128.9$ $\left(\mathrm{CH}_{\mathrm{AR}}\right), 129.4\left(\mathrm{CH}_{\mathrm{AR}}\right), 129.6\left(\mathrm{CH}_{\mathrm{AR}}\right), 130.7\left(\mathrm{C}_{\mathrm{AR}}\right), 134.5\left(\mathrm{CH}_{\mathrm{AR}}\right), 135.6\left(\mathrm{C}-3, \mathrm{C}_{\mathrm{AR}}\right), 136.2(\mathrm{C}-4), 144.9$ $\left(\mathrm{CH}_{\mathrm{AR}}\right), 161.1$ (NCO); HRMS (ESI) calcd for $\left[\mathrm{C}_{18} \mathrm{H}_{17} \mathrm{NO}_{3} \mathrm{~S}_{2}+\mathrm{H}^{+}\right]$: 360.0723 , found: 360.0720 .

1-(tert-Butoxycarbonyl)-3-(phenylsulfinyl)-5,6-dihydro-2(1H)-pyridone (5a). $m$-Chloroperbenzoic acid ( $1.51 \mathrm{~g}$, technical grade $70 \%, 1.06 \mathrm{~g}$ pure oxidant, $6.14 \mathrm{mmol})$ in $\mathrm{CH}_{2} \mathrm{Cl}_{2}(100 \mathrm{~mL})$ was added to a solution of phenylthio derivative $4 \mathrm{a}(1.884 \mathrm{~g}, 6.14 \mathrm{mmol})$ in $\mathrm{CH}_{2} \mathrm{Cl}_{2}(200 \mathrm{~mL})$ at $-78^{\circ} \mathrm{C}$. The solution was stirred at this temperature for $1.5 \mathrm{~h}$. Then, a saturated solution of $\mathrm{NaHCO}_{3}$ was added, the mixture was extracted with $\mathrm{CH}_{2} \mathrm{Cl}_{2}$, and the organic solvent was removed under reduced pressure. The crude product was purified by flash chromatography (95:5 hexane-EtOAc) to afford sulfinyl derivative $\mathbf{5 a}$ (1.39 g, 70\% yield): IR (ATR Pike) $v\left(\mathrm{~cm}^{-1}\right)$ : 1762 (CO), 1719 (CO), 1099, 1049 (SO); ${ }^{1} \mathrm{H}-\mathrm{NMR}(400 \mathrm{MHz}$, $\left.\mathrm{CDCl}_{3}, \mathrm{COSY}, \mathrm{g}-\mathrm{HSQC}\right): \delta=1.51\left[\mathrm{~s}, 9 \mathrm{H},\left(\mathrm{CH}_{3}\right)_{3} \mathrm{C}\right], 2.55-2.75(\mathrm{~m}, 2 \mathrm{H}, \mathrm{H}-5), 3.59(\mathrm{ddd}, J=13.2,10.4$, $5.2 \mathrm{~Hz}, 1 \mathrm{H}, \mathrm{H}-6), 4.05(\mathrm{dtd}, J=13.2,5.2,1.1 \mathrm{~Hz}, \mathrm{H}-6), 7.45-7.47\left(\mathrm{~m}, 3 \mathrm{H}, \mathrm{H}_{\mathrm{AR}}\right), 7.57(\mathrm{td}, J=3.6,1.2 \mathrm{~Hz}$, $1 \mathrm{H}, \mathrm{H}-4), 7.79-7.81\left(\mathrm{~m}, 2 \mathrm{H}, \mathrm{H}_{\mathrm{AR}}\right) ;{ }^{13} \mathrm{C}-\mathrm{NMR}\left(100.6 \mathrm{MHz}, \mathrm{CDCl}_{3}\right): \delta=24.9(\mathrm{C}-5), 27.9\left[\left(\mathrm{CH}_{3}\right)_{3} \mathrm{C}\right], 43.2$ $\left.(\mathrm{C}-6), 83.8\left[\left(\mathrm{CH}_{3}\right)_{3} \mathrm{C}\right)\right], 125.6\left(2 \mathrm{CH}_{\mathrm{AR}}\right), 129.1\left(2 \mathrm{CH}_{\mathrm{AR}}\right), 131.3\left(\mathrm{CH}_{\mathrm{AR}}\right), 142.3(\mathrm{C}-4), 142.6,143.7\left(\mathrm{C}_{\mathrm{AR}}, \mathrm{C}-3\right)$, 151.9 (CO), 160.2 (CO); HRMS (ESI) calcd for $\left[\mathrm{C}_{16} \mathrm{H}_{19} \mathrm{NO}_{4} \mathrm{~S}+\mathrm{Na}^{+}\right]$: 344.0927, found: 344.0927.

1-(Methyl)-3-(phenylsulfinyl)-5,6-dihydro-2(1H)-pyridone (5b). Operating as described for the preparation of compound $5 \mathbf{a}$, from a solution of phenylthio derivative $4 \mathbf{b}(163 \mathrm{mg}, 0.74 \mathrm{mmol})$ in $\mathrm{CH}_{2} \mathrm{Cl}_{2}(15 \mathrm{~mL})$ and $m$-CPBA (183 mg, technical grade $70 \%, 0.74 \mathrm{mmol})$ in $\mathrm{CH}_{2} \mathrm{Cl}_{2}(20 \mathrm{~mL})$, sulfinyl derivative $5 \mathbf{b}$ was obtained (100 mg, 57\% yield): IR (ATR Pike) $v\left(\mathrm{~cm}^{-1}\right): 1652$ (CO), 1043 (SO); ${ }^{1} \mathrm{H}-\mathrm{NMR}(400 \mathrm{MHz}$, $\mathrm{CDCl}_{3}, \mathrm{COSY}$, g-HSQC): $\delta=2.53-2.74(2 \mathrm{~m}, 2 \mathrm{H}, \mathrm{H}-5), 2.90\left(\mathrm{~s}, 3 \mathrm{H}, \mathrm{CH}_{3}\right), 3.38-3.46(\mathrm{dd}, J=8.8,6.8 \mathrm{~Hz}$, $2 \mathrm{H}, \mathrm{H}-6), 7.31(\mathrm{t}, J=4.8 \mathrm{~Hz}, 1 \mathrm{H}, \mathrm{H}-4), 7.44-7.45\left(\mathrm{~m}, 3 \mathrm{H}, \mathrm{H}_{\mathrm{AR}}\right), 7.78-7.81\left(\mathrm{~m}, 2 \mathrm{H}, \mathrm{H}_{\mathrm{AR}}\right) ;{ }^{13} \mathrm{C}-\mathrm{NMR}$ $\left(100.6 \mathrm{MHz}, \mathrm{CDCl}_{3}\right): \delta=24.0(\mathrm{C}-5), 33.7\left(\mathrm{CH}_{3}\right), 46.8(\mathrm{C}-6), 125.5\left(2 \mathrm{CH}_{\mathrm{AR}}\right), 128.8\left(2 \mathrm{CH}_{\mathrm{AR}}\right), 131.0\left(\mathrm{CH}_{\mathrm{AR}}\right)$, $138.1(\mathrm{C}-4), 140.6,143.8\left(\mathrm{C}_{\mathrm{AR}}, \mathrm{C}-3\right), 160.1$ (NCO); HRMS (ESI) calcd for $\left[\mathrm{C}_{12} \mathrm{H}_{13} \mathrm{NO}_{2} \mathrm{~S}+\mathrm{H}^{+}\right]: 236.074$, found: 236.0741 .

3-(Phenylsulfinyl)-1-(p-toluenesulfonyl)-5,6-dihydro-2(1H)-pyridone (5c). Operating as described for the preparation of compound $5 \mathbf{a}$, from a solution of phenylthio derivative $4 \mathbf{c}(55 \mathrm{mg}, 0.12 \mathrm{mmol})$ in $\mathrm{CH}_{2} \mathrm{Cl}_{2}$ (3 mL) and $m$-CPBA (30 mg, technical grade $70 \%, 0.12 \mathrm{mmol}$ ) in $\mathrm{CH}_{2} \mathrm{Cl}_{2}(3 \mathrm{~mL})$, sulfinyl derivative $5 \mathbf{c}$ was obtained. This compound was used in the next step without further purification: IR (ATR Pike) $v$ ( $\mathrm{cm}^{-1}$ ): 1683, (NCO), 1362 (SO2), 1170 (SO); ${ }^{1} \mathrm{H}-\mathrm{NMR}\left(400 \mathrm{MHz}, \mathrm{CDCl}_{3}, \mathrm{COSY}, \mathrm{g}-\mathrm{HSQC}\right): \delta=2.43$ (s, $\left.3 \mathrm{H}, \mathrm{CH}_{3}\right), 2.67-2.81(\mathrm{~m}, 2 \mathrm{H}, \mathrm{H}-5), 3.78-3.86(\mathrm{~m}, 1 \mathrm{H}, \mathrm{H}-6), 4.11-4.20(\mathrm{~m}, 1 \mathrm{H}, \mathrm{H}-6), 7.27(\mathrm{~d}, J=7.6 \mathrm{~Hz}$, $\left.2 \mathrm{H}, \mathrm{H}_{\mathrm{AR}}\right), 7.36-7.41\left(\mathrm{~m}, 3 \mathrm{H}, \mathrm{H}_{\mathrm{AR}}\right), 7.52(\mathrm{dd}, J=4.4,3.6 \mathrm{~Hz}, 1 \mathrm{H}, \mathrm{H}-4), 7.63-7.66\left(\mathrm{~m}, 2 \mathrm{H}, \mathrm{H}_{\mathrm{AR}}\right), 7.82$ $\left(\mathrm{d}, J=8.0 \mathrm{~Hz}, 2 \mathrm{H}, \mathrm{H}_{\mathrm{AR}}\right) ;{ }^{13} \mathrm{C}-\mathrm{NMR}\left(100.6 \mathrm{MHz}, \mathrm{CDCl}_{3}\right): \delta=21.6\left(\mathrm{CH}_{3}\right), 25.3(\mathrm{C}-5), 43.8(\mathrm{C}-6), 125.1$ $\left(2 \mathrm{CH}_{\mathrm{AR}}\right), 128.3\left(2 \mathrm{CH}_{\mathrm{AR}}\right), 129.1\left(2 \mathrm{CH}_{\mathrm{AR}}\right), 129.4\left(2 \mathrm{CH}_{\mathrm{AR}}\right), 131.3\left(\mathrm{CH}_{\mathrm{AR}}\right), 135.1\left(\mathrm{C}_{\mathrm{AR}}\right), 142.8(\mathrm{C}-4), 143.2$, $145.1\left(\mathrm{C}_{\mathrm{AR}}, \mathrm{C}-3\right), 159.7(\mathrm{NCO})$.

1-(tert-Butoxycarbonyl)-3-(phenylsulfonyl)-5,6-dihydro-2(1H)-pyridone (6a). $m$-Chloroperbenzoic acid (1.06 mg, technical grade 70\%, $745 \mathrm{mg}$ pure oxidant, $4.32 \mathrm{mmol})$ in $\mathrm{CH}_{2} \mathrm{Cl}_{2}(150 \mathrm{~mL})$ was added to a solution of sulfinyl derivative $5 \mathrm{a}(1.39 \mathrm{~g}, 4.32 \mathrm{mmol})$ in $\mathrm{CH}_{2} \mathrm{Cl}_{2}(60 \mathrm{~mL})$ at $-78^{\circ} \mathrm{C}$. The solution was stirred at room temperature for $6 \mathrm{~h}$. Then, a saturated solution of $\mathrm{NaHCO}_{3}$ was added, the mixture was extracted with $\mathrm{CH}_{2} \mathrm{Cl}_{2}$, and the organic solvent was removed under reduced pressure to afford sulfonyl derivative $6 \mathbf{a}$, which was used in the next step without purification: IR (ATR Pike) $v\left(\mathrm{~cm}^{-1}\right)$ : 1721 (CO), 1689 (CO), $1446\left(\mathrm{SO}_{2}\right), 1143\left(\mathrm{SO}_{2}\right) ;{ }^{1} \mathrm{H}-\mathrm{NMR}\left(400 \mathrm{MHz}, \mathrm{CDCl}_{3}, \mathrm{COSY}, \mathrm{g}-\mathrm{HSQC}\right): \delta=1.49$ [s, $9 \mathrm{H},\left(\mathrm{CH}_{3}\right)_{3} \mathrm{Cl}, 2.67(\mathrm{td}, J=6.0,4.0 \mathrm{~Hz}, 2 \mathrm{H}, \mathrm{H}-5), 3.87(\mathrm{t}, J=6.8 \mathrm{~Hz}, 2 \mathrm{H}, \mathrm{H}-6), 7.51-7.53\left(\mathrm{~m}, 2 \mathrm{H}, \mathrm{H}_{\mathrm{AR}}\right)$, $7.60\left(\mathrm{t}, J=7.6 \mathrm{~Hz}, 1 \mathrm{H}, \mathrm{H}_{\mathrm{AR}}\right), 8.05\left(\mathrm{dm}, J=7.2 \mathrm{~Hz}, 2 \mathrm{H}, \mathrm{H}_{\mathrm{AR}}\right), 8.08(\mathrm{t}, J=4.4 \mathrm{~Hz}, 1 \mathrm{H}, \mathrm{H}-4) ;{ }^{13} \mathrm{C}-\mathrm{NMR}$ $\left(100.6 \mathrm{MHz}, \mathrm{CDCl}_{3}\right): \delta=25.0(\mathrm{C}-5), 27.9\left[\left(\mathrm{CH}_{3}\right)_{3} \mathrm{C}\right], 42.9(\mathrm{C}-6), 84.2\left[\left(\mathrm{CH}_{3}\right)_{3} \mathrm{C}\right], 128.7\left(2 \mathrm{CH}_{\mathrm{AR}}\right), 129.3$ $\left(2 \mathrm{CH}_{\mathrm{AR}}\right), 133.5\left(\mathrm{CH}_{\mathrm{AR}}\right), 138.7,139.5\left(\mathrm{C}-3, \mathrm{C}_{\mathrm{AR}}\right), 151.4(\mathrm{CO}), 152.2(\mathrm{C}-4), 157.8$ (CO); HRMS (ESI) calcd for $\left[\mathrm{C}_{16} \mathrm{H}_{19} \mathrm{NO}_{5} \mathrm{~S}+\mathrm{H}^{+}\right]$: 338.1057, found: 338.1066 . 
1-Methyl-3-(phenylsulfonyl)-5,6-dihydro-2(1H)-pyridone (6b). Operating as described for the preparation of compound $\mathbf{6 a}$ (reaction time $20 \mathrm{~h}$ ), from a solution of sulfinyl derivative $5 \mathbf{b}$ (100 $\mathrm{mg}, 0.42 \mathrm{mmol}$ ) in $\mathrm{CH}_{2} \mathrm{Cl}_{2}(10 \mathrm{~mL})$ and $m$-CPBA (104 mg, technical grade $\left.70 \%, 0.42 \mathrm{mmol}\right)$ in $\mathrm{CH}_{2} \mathrm{Cl}_{2}(10 \mathrm{~mL})$, sulfonyl derivative $6 \mathbf{b}$ was obtained. This compound was used in the next step without purification: IR (ATR Pike) $v\left(\mathrm{~cm}^{-1}\right)$ : $1662(\mathrm{NCO}), 1306\left(\mathrm{SO}_{2}\right), 1151\left(\mathrm{SO}_{2}\right) ;{ }^{1} \mathrm{H}-\mathrm{NMR}\left(400 \mathrm{MHz}, \mathrm{CDCl}_{3}, \mathrm{COSY}, \mathrm{g}-\mathrm{HSQC}\right)$ : $\delta=2.67(\mathrm{td}, J=7.2,4.4 \mathrm{~Hz}, 2 \mathrm{H}, \mathrm{H}-5), 2.93\left(\mathrm{~s}, 3 \mathrm{H}, \mathrm{CH}_{3}\right), 3.46(\mathrm{t}, J=7.2 \mathrm{~Hz}, 2 \mathrm{H}, \mathrm{H}-6), 7.51(\mathrm{tm}, J=7.6 \mathrm{~Hz}$, $\left.2 \mathrm{H}, \mathrm{H}_{\mathrm{AR}}\right), 7.57\left(\mathrm{~m}, 1 \mathrm{H}, \mathrm{H}_{\mathrm{AR}}\right), 7.87(\mathrm{t}, J=4.4 \mathrm{~Hz}, 1 \mathrm{H}, \mathrm{H}-4), 8.05\left(\mathrm{dm}, J=7.6 \mathrm{~Hz}, 2 \mathrm{H}, \mathrm{H}_{\mathrm{AR}}\right) ;{ }^{13} \mathrm{C}-\mathrm{NMR}$ $\left(100.6 \mathrm{MHz}, \mathrm{CDCl}_{3}\right): \delta=24.3(\mathrm{C}-5), 34.4\left(\mathrm{CH}_{3}\right), 46.6(\mathrm{C}-6), 128.6\left(2 \mathrm{CH}_{\mathrm{AR}}\right), 129.1\left(2 \mathrm{CH}_{\mathrm{AR}}\right), 133.4$ $\left(\mathrm{CH}_{\mathrm{AR}}\right), 140.0\left(\mathrm{C}-3, \mathrm{C}_{\mathrm{AR}}\right), 148.4(\mathrm{C}-4), 159.0(\mathrm{CO})$; HRMS (ESI) calcd for $\left[\mathrm{C}_{12} \mathrm{H}_{13} \mathrm{NO}_{3} \mathrm{~S}+\mathrm{H}^{+}\right]: 252.0689$, found: 252.0679 .

3-(Phenylsulfonyl)-1-(p-toluenesulfonyl)-5,6-dihydro-2(1H)-pyridone (6c). Operating as described for the preparation of compound 6a (reaction time $20 \mathrm{~h}$ ), from a solution of sulfinyl derivative $5 \mathrm{c}(45 \mathrm{mg}$, $0.12 \mathrm{mmol}$ ) in $\mathrm{CH}_{2} \mathrm{Cl}_{2}(6 \mathrm{~mL})$ and $m$-CPBA ( $30 \mathrm{mg}$, technical grade $70 \% ; 21 \mathrm{mg}$ pure oxidant, $0.12 \mathrm{mmol}$ ) in $\mathrm{CH}_{2} \mathrm{Cl}_{2}(6 \mathrm{~mL})$, sulfonyl derivative $\mathbf{6 c}$ was obtained. This compound was used in the next step without purification: IR (ATR Pike) $v\left(\mathrm{~cm}^{-1}\right): 1695(\mathrm{NCO}), 1362\left(\mathrm{SO}_{2}\right), 1157\left(\mathrm{SO}_{2}\right) ;{ }^{1} \mathrm{H}-\mathrm{NMR}(400 \mathrm{MHz}$, $\mathrm{CDCl}_{3}, \mathrm{COSY}$, g-HSQC): $\delta=2.42\left(\mathrm{~s}, 1 \mathrm{H}, \mathrm{CH}_{3}\right), 2.79(\mathrm{td}, J=6.4,4.4 \mathrm{~Hz}, 2 \mathrm{H}, \mathrm{H}-5), 4.04(\mathrm{t}, J=6.0 \mathrm{~Hz}, 2 \mathrm{H}$, $\mathrm{H}-6), 7.29\left(\mathrm{~d}, J=8.0 \mathrm{~Hz}, 2 \mathrm{H}, \mathrm{H}_{\mathrm{AR}}\right), 7.47-7.51\left(\mathrm{~m}, 2 \mathrm{H}, \mathrm{H}_{\mathrm{AR}}\right), 7.57\left(\mathrm{tt}, J=7.6,1.2 \mathrm{~Hz}, 1 \mathrm{H}, \mathrm{H}_{\mathrm{AR}}\right), 7.85(\mathrm{~d}$, $\left.J=8.0 \mathrm{~Hz}, 2 \mathrm{H}, \mathrm{H}_{\mathrm{AR}}\right), 7.96\left(\mathrm{~d}, J=8.0 \mathrm{~Hz}, 2 \mathrm{H}, \mathrm{H}_{\mathrm{AR}}\right), 8.06(\mathrm{t}, J=4.4 \mathrm{~Hz}, 1 \mathrm{H}, \mathrm{H}-4) ;{ }^{13} \mathrm{C}-\mathrm{NMR}(100.6 \mathrm{MHz}$, $\left.\mathrm{CDCl}_{3}\right): \delta=21.6\left(\mathrm{CH}_{3}\right), 25.7(\mathrm{C}-5), 43.5(\mathrm{C}-6), 128.4\left(2 \mathrm{CH}_{\mathrm{AR}}\right), 128.8\left(2 \mathrm{CH}_{\mathrm{AR}}\right), 129.1\left(2 \mathrm{CH}_{\mathrm{AR}}\right), 129.6$ $\left(2 \mathrm{CH}_{\mathrm{AR}}\right), 133.7\left(\mathrm{CH}_{\mathrm{AR}}\right), 134.9\left(\mathrm{C}_{\mathrm{AR}}\right), 137.8\left(\mathrm{CH}_{\mathrm{AR}}\right), 139.0(\mathrm{C}-3), 145.3\left(\mathrm{C}_{\mathrm{AR}}\right), 153.2(\mathrm{C}-4), 157.2(\mathrm{NCO})$.

1-(tert-Butoxycarbonyl)-3-(ethoxycarbonyl)-3-(phenylselenyl)-2-piperidone (7). Lithium bis(trimethyl silyl)amide (11.1 mL of a $1 \mathrm{M}$ solution in THF, $11.1 \mathrm{mmol}$ ) was slowly added at $-78{ }^{\circ} \mathrm{C}$ under an argon atmosphere to a solution of lactam $\mathbf{1 b}(1.0 \mathrm{~g}, 5.05 \mathrm{mmol})$ in anhydrous THF $(100 \mathrm{~mL})$, and the solution was stirred for $1 \mathrm{~h}$. Then, ethyl chloroformate $(532 \mu \mathrm{l}, 5.56 \mathrm{mmol})$ and, after $1 \mathrm{~h}$ of continuous stirring at $-78{ }^{\circ} \mathrm{C}$, a solution of $\mathrm{PhSeCl}(1.48 \mathrm{~g}, 7.58 \mathrm{mmol})$ in anhydrous THF $(10 \mathrm{~mL})$ were added to the solution. The mixture was stirred for a further $1 \mathrm{~h}$ and poured into saturated aqueous $\mathrm{NH}_{4} \mathrm{Cl}$. The resulting mixture was extracted with EtOAc, and the combined organic extracts were dried, filtered, and concentrated under reduced pressure. Flash chromatography ( $8: 2$ hexane-EtOAc) of the resulting oil gave the seleno derivative $7\left(1.79 \mathrm{~g}, 83 \%\right.$ yield) as a yellow foam: IR (ATR Pike) $v\left(\mathrm{~cm}^{-1}\right)$ : $1718(\mathrm{CO}) ;{ }^{1} \mathrm{H}-\mathrm{NMR}\left(300 \mathrm{MHz}, \mathrm{CDCl}_{3}\right): \delta=1.27\left(\mathrm{t}, J=7.2 \mathrm{~Hz}, 3 \mathrm{H}, \mathrm{CH}_{2} \mathrm{CH}_{3}\right), 1.54\left[\mathrm{~s}, 9 \mathrm{H},\left(\mathrm{CH}_{3}\right)_{3} \mathrm{C}\right], 1.73$ (m, 1H, H-5), $1.84(\mathrm{~m}, 1 \mathrm{H}, \mathrm{H}-5), 2.01$ (ddd, $J=13.8,10.2,5.7 \mathrm{~Hz}, 1 \mathrm{H}, \mathrm{H}-4), 2.28(\mathrm{dt}, J=13.8,6.0 \mathrm{~Hz}, 1 \mathrm{H}$, $\mathrm{H}-4), 3.52(\mathrm{~m}, 1 \mathrm{H}, \mathrm{H}-6), 3.61$ (ddd, $J=13.2,8.4,5.4 \mathrm{~Hz}, 1 \mathrm{H}, \mathrm{H}-6), 4.22$ (qd, $J=7.2,3.3 \mathrm{~Hz}, 2 \mathrm{H}, \mathrm{CH}_{2} \mathrm{CH}_{3}$ ), $7.31\left(\mathrm{t}, J=7.2,2 \mathrm{H}, \mathrm{H}_{\mathrm{AR}}\right), 7.41\left(\mathrm{t}, J=7.2 \mathrm{~Hz}, 1 \mathrm{H}, \mathrm{H}_{\mathrm{AR}}\right), 7.65\left(\mathrm{t}, J=7.2 \mathrm{~Hz}, 2 \mathrm{H}, \mathrm{H}_{\mathrm{AR}}\right) ; \mathrm{HRMS}$ (ESI) calcd for $\left[\mathrm{C}_{19} \mathrm{H}_{25} \mathrm{NO}_{5} \mathrm{Se}+\mathrm{Na}^{+}\right]$: 450.0787, found: 450.0788 .

1-(tert-Butoxycarbonyl)-3-(ethoxycarbonyl)-5,6-dihydro-2(1H)-pyridone (8). $\mathrm{H}_{2} \mathrm{O}_{2}(900 \mu \mathrm{l}, 29.35 \mathrm{mmol}$ ) and pyridine ( $405 \mu \mathrm{l}, 5.03 \mathrm{mmol})$ were added at room temperature to a solution of seleno lactam $7(1.79 \mathrm{~g}$, $4.19 \mathrm{mmol})$ in $\mathrm{CH}_{2} \mathrm{Cl}_{2}(300 \mathrm{~mL})$, and the resulting solution was stirred at room temperature for 30 minutes. Water was then added, and the mixture was extracted with $\mathrm{CH}_{2} \mathrm{Cl}_{2}$. The combined organic extracts were dried, filtered, and concentrated under reduced pressure. Flash chromatography (8:2 hexane-EtOAc) of the resulting oil gave unsaturated lactam 8 (981 mg, 87\% yield) as a yellow foam: IR (ATR Pike) $v\left(\mathrm{~cm}^{-1}\right): 1715(\mathrm{CO}) ;{ }^{1} \mathrm{H}-\mathrm{NMR}\left(400 \mathrm{MHz}, \mathrm{CDCl}_{3}, \mathrm{COSY}, \mathrm{g}-\mathrm{HSQC}\right): \delta=1.33(\mathrm{t}, J=6.8 \mathrm{~Hz}$, $\left.3 \mathrm{H}, \mathrm{CH}_{2} \mathrm{CH}_{3}\right), 1.54$ [s, 9H, $\left.\left(\mathrm{CH}_{3}\right)_{3} \mathrm{C}\right], 2.52(\mathrm{td}, J=6.4,4.4 \mathrm{~Hz}, 2 \mathrm{H}, \mathrm{H}-5), 3.88(\mathrm{t}, J=6.4 \mathrm{~Hz}, 2 \mathrm{H}, \mathrm{H}-6)$, $4.29\left(\mathrm{q}, J=6.8 \mathrm{~Hz}, 2 \mathrm{H}, \mathrm{CH}_{2} \mathrm{CH}_{3}\right), 7.48(\mathrm{t}, J=.4 \mathrm{~Hz}, 1 \mathrm{H}, \mathrm{H}-4) ;{ }^{13} \mathrm{C}-\mathrm{NMR}\left(100.6 \mathrm{MHz}, \mathrm{CDCl}_{3}\right): \delta=14.1$ $\left(\mathrm{CH}_{2} \mathrm{CH}_{3}\right), 24.7(\mathrm{C}-5), 28.0\left[\left(\mathrm{CH}_{3}\right)_{3} \mathrm{C}\right], 43.0(\mathrm{C}-6), 61.5\left(\mathrm{CH}_{2} \mathrm{CH}_{3}\right), 83.6\left[\left(\mathrm{CH}_{3}\right)_{3} \mathrm{C}\right], 130.9(\mathrm{C}-3), 148.5(\mathrm{C}-4)$, 160.0 (CO), 164.0 (CO); HRMS (ESI) calcd for $\left[\mathrm{C}_{13} \mathrm{H}_{19} \mathrm{NO}_{5}+\mathrm{Na}^{+}\right]$: 292.1155, found: 292.1166.

\subsection{General Procedure for the Double Michael Addition Reactions}

A solution of unsaturated lactam $\mathbf{6 a}, \mathbf{b}, \mathbf{c}$ or 8 (1 equiv.) in anhydrous $\mathrm{CH}_{2} \mathrm{Cl}_{2}, \mathrm{MeCN}$ or $\mathrm{MeOH}$ was added at $0{ }^{\circ} \mathrm{C}$ under an argon atmosphere to a solution of the Nazarov reagent ( 9 or 15) and $\mathrm{Cs}_{2} \mathrm{CO}_{3}$ 
or $\mathrm{KF}$ in anhydrous $\mathrm{CH}_{2} \mathrm{Cl}_{2}, \mathrm{MeCN}$ or $\mathrm{MeOH}$, and the resulting mixture was allowed to warm slowly to room temperature. After $20 \mathrm{~h}$ of stirring at room temperature, the mixture was concentrated under reduced pressure. Flash chromatography (9:1 hexane-EtOAc) of the residue afforded the corresponding adduct/s (10a,b, 12a,b, 16a, b,c or 17) as yellow foams (detailed data in supplementary materials).

cis-2-(tert-Butoxycarbonyl)-6-hydroxy-5-(methoxycarbonyl)-8-methyl-1-oxo-8a-(phenylsulfonyl)-1,2,3,4,4a, $7,8,8 a$-octahydroisoquinolines $\mathbf{1 0 a}$ and $\mathbf{1 0 b}$. Operating as described above in the general procedure, from unsaturated lactam $6 \mathbf{a}(800 \mathrm{mg}, 2.28 \mathrm{mmol})$ in anhydrous $\mathrm{CH}_{2} \mathrm{Cl}_{2}(200 \mathrm{~mL})$, Nazarov reagent 9 (1.07 $\mathrm{g}$, $7.5 \mathrm{mmol})$, and $\mathrm{Cs}_{2} \mathrm{CO}_{3}(4.9 \mathrm{~g}, 15 \mathrm{mmol})$ in anhydrous $\mathrm{CH}_{2} \mathrm{Cl}_{2}(250 \mathrm{~mL})$, cis-hydroisoquinolones 10a (cis $\mathrm{Me} / \mathrm{SO}_{2} \mathrm{Ph}$ isomer) and $\mathbf{1 0 b}$ (trans $\mathrm{Me} / \mathrm{SO}_{2} \mathrm{Ph}$ isomer) were obtained (ratio 3:1, $890 \mathrm{mg}, 79 \%$ yield). Compound 10a: IR (ATR Pike) $v\left(\mathrm{~cm}^{-1}\right)$ : 1727 (CO), 1654 (CO); ${ }^{1} \mathrm{H}-\mathrm{NMR}\left(400 \mathrm{MHz}, \mathrm{CDCl}_{3}\right.$, COSY, g-HSQC): $\delta=1.08\left(\mathrm{~d}, J=6.4 \mathrm{~Hz}, 3 \mathrm{H}, \mathrm{CH}_{3}\right), 1.50$ [s, 9H, $\left.\left(\mathrm{CH}_{3}\right)_{3} \mathrm{C}\right], 1.89(\mathrm{~m}, 1 \mathrm{H}, \mathrm{H}-7), 2.32(\mathrm{dm}$, $J=14.8 \mathrm{~Hz}, 1 \mathrm{H}, \mathrm{H}-4), 2.40$ (m, 2H, H-8, H-7), 2.70 (dddd, $J=14.8,13.6,5.2,3.6 \mathrm{~Hz}, 1 \mathrm{H}, \mathrm{H}-4), 3.23$ (td, $J=12.8,4.0 \mathrm{~Hz}, 1 \mathrm{H}, \mathrm{H}-3), 3.74(\mathrm{dm}, J=12.8 \mathrm{~Hz}, 1 \mathrm{H}, \mathrm{H}-3), 3.82$ (s, 3H, CH $3 \mathrm{O}) 3.92$ (br. s, 1H, H-4a), $7.49\left(\mathrm{td}, J=7.6,2.0 \mathrm{~Hz}, 2 \mathrm{H}, \mathrm{H}_{\mathrm{AR}}\right), 7.63\left(\mathrm{td}, J=7.6,1.2 \mathrm{~Hz}, 1 \mathrm{H}, \mathrm{H}_{\mathrm{AR}}\right), 7.96-7.99(\mathrm{dd}, J=7.6,1.2 \mathrm{~Hz}$, $\left.2 \mathrm{H}, \mathrm{H}_{\mathrm{AR}}\right) ;{ }^{13} \mathrm{C}-\mathrm{NMR}\left(100.6 \mathrm{MHz}, \mathrm{CDCl}_{3}\right): \delta=14.0\left(\mathrm{CH}_{3}\right), 23.3(\mathrm{C}-4), 27.8\left[\left(\mathrm{CH}_{3}\right)_{3} \mathrm{C}\right], 29.3(\mathrm{C}-4 \mathrm{a}), 30.8$ (C-8), $35.0(\mathrm{C}-7), 42.9(\mathrm{C}-3), 51.8\left(\mathrm{CH}_{3} \mathrm{O}\right), 77.4(\mathrm{C}-8 \mathrm{a}), 83.6\left[\left(\mathrm{CH}_{3}\right)_{3} \mathrm{C}\right], 96.6(\mathrm{C}-5), 128.3\left(2 \mathrm{CH}_{\mathrm{AR}}\right), 130.9$ $\left(2 \mathrm{CH}_{\mathrm{AR}}\right), 134.1\left(\mathrm{CH}_{\mathrm{AR}}\right), 137.0\left(\mathrm{C}_{\mathrm{AR}}\right), 151.8(\mathrm{CO}), 167.1(\mathrm{CO}), 171.5,171.6(\mathrm{CO}, \mathrm{C}-6)$; HRMS (ESI) calcd for $\left[\mathrm{C}_{23} \mathrm{H}_{29} \mathrm{NO}_{8} \mathrm{~S}+\mathrm{Na}^{+}\right]$: 502.1506, found: 502.1511. Compound 10b: ${ }^{1} \mathrm{H}-\mathrm{NMR}\left(400 \mathrm{MHz}, \mathrm{CDCl}_{3}\right.$, COSY, g-HSQC): $\delta=1.06\left(\mathrm{~d}, J=6.8 \mathrm{~Hz}, 3 \mathrm{H}, \mathrm{CH}_{3}\right), 1.53\left[\mathrm{~s}, 9 \mathrm{H},\left(\mathrm{CH}_{3}\right)_{3} \mathrm{C}\right], 1.69(\mathrm{ddd}, J=13.2,3.6,1 \mathrm{H}$, H-4), 2.17 (d, J = 18.4 Hz, 1H, H-7), 2.44 (dd, J = 14.0, 2.8 Hz, 1H, H-4), 2.79 (m, 1H, H-8), 3.15 (dd, $J=18.0,6.0 \mathrm{~Hz}, 1 \mathrm{H}, \mathrm{H}-7), 3.39(\mathrm{td}, J=12.8,3.2 \mathrm{~Hz}, 1 \mathrm{H}, \mathrm{H}-3), 3.71(\mathrm{dd}, J=12.8,1.2,1 \mathrm{H}, \mathrm{H}-4 \mathrm{a}), 3.86(\mathrm{~s}, 3 \mathrm{H}$, $\left.\mathrm{CH}_{3} \mathrm{O}\right) 3.94(\mathrm{dt}, J=12.4,2.4 \mathrm{~Hz}, \mathrm{H}-3), 7.54\left(\mathrm{t}, J=7.2 \mathrm{~Hz}, 2 \mathrm{H}, \mathrm{H}_{\mathrm{AR}}\right), 7.68\left(\mathrm{td}, J=8.0,1.2 \mathrm{~Hz}, 1 \mathrm{H}, \mathrm{H}_{\mathrm{AR}}\right)$, $7.89\left(\mathrm{~d}, J=8.0 \mathrm{~Hz}, 2 \mathrm{H}, \mathrm{H}_{\mathrm{AR}}\right) ;{ }^{13} \mathrm{C}-\mathrm{NMR}\left(100.6 \mathrm{MHz}, \mathrm{CDCl}_{3}\right): \delta=20.4\left(\mathrm{CH}_{3}\right), 27.7(\mathrm{C}-4), 27.9\left[\left(\mathrm{CH}_{3}\right)_{3} \mathrm{C}\right]$, 30.1 (C-8), 33.2 (C-7), 33.2 (C-4a), $45.7(\mathrm{C}-3), 51.8\left(\mathrm{CH}_{3} \mathrm{O}\right), 77.9(\mathrm{C}-8 \mathrm{a}), 83.5\left[\left(\mathrm{CH}_{3}\right)_{3} \mathrm{C}\right], 96.8(\mathrm{C}-5), 128.5$ $\left(2 \mathrm{CH}_{\mathrm{AR}}\right), 131.0\left(2 \mathrm{CH}_{\mathrm{AR}}\right), 134.3\left(\mathrm{CH}_{\mathrm{AR}}\right), 134.9\left(\mathrm{C}_{\mathrm{AR}}\right), 151.5(\mathrm{CO}), 166.3(\mathrm{CO}), 171.0,171.8(\mathrm{CO}, \mathrm{C}-6)$.

cis-6-Hydroxy-5-(methoxycarbonyl)-2,8-dimethyl-1-oxo-8a-(phenylsulfonyl)-1,2,3,4,4a,7,8,8a-octahydroisoquinolines $\mathbf{1 2 a}$ and $\mathbf{1 2 b}$. Operating as described in the general procedure, from unsaturated lactam $\mathbf{6 b}$ $(220 \mathrm{mg}, 0.87 \mathrm{mmol})$ in anhydrous $\mathrm{MeOH}(160 \mathrm{~mL})$, Nazarov reagent 9 (372 mg, $2.62 \mathrm{mmol})$, and $\mathrm{KF}$ (304 mg, $5.24 \mathrm{mmol})$ in anhydrous $\mathrm{MeOH}(10 \mathrm{~mL})$, cis-hydroisoquinolones 12a (cis $\mathrm{Me} / \mathrm{SO}_{2} \mathrm{Ph}$ isomer) and $\mathbf{1 2 b}$ (trans $\mathrm{Me} / \mathrm{SO}_{2} \mathrm{Ph}$ isomer) were obtained (ratio 1:5, $175 \mathrm{mg}, 51 \%$ yield). Compound 12a: IR (ATR Pike) $v\left(\mathrm{~cm}^{-1}\right): 1646(\mathrm{CO}) ;{ }^{1} \mathrm{H}-\mathrm{NMR}\left(400 \mathrm{MHz}, \mathrm{CDCl}_{3}, \mathrm{COSY}, \mathrm{g}-\mathrm{HSQC}\right): \delta=1.09(\mathrm{~d}, \mathrm{~J}=6.8 \mathrm{~Hz}$, $\left.3 \mathrm{H}, \mathrm{CH}_{3}\right), 1.90(\mathrm{dd}, J=18.5,2.0 \mathrm{~Hz}, 1 \mathrm{H}, \mathrm{H}-7), 2.30(\mathrm{dq}, J=17.6,2.4 \mathrm{~Hz}, 1 \mathrm{H}, \mathrm{H}-4), 2.39(\mathrm{dm}, J=18.5 \mathrm{~Hz}$, 1H, H-7), 2.48 (m, 1H, H-8), 2.67-2.73 (m, 1H, H-4), 2.96 (s, 3H, CH $\mathrm{CH}_{3}$ ), 3.21 (m, 2H, H-3), 3.84 (s, 3H, $\left.\mathrm{CH}_{3} \mathrm{O}\right), 7.53\left(\mathrm{t}, J=7.6 \mathrm{~Hz}, 2 \mathrm{H}, \mathrm{H}_{\mathrm{AR}}\right), 7.65\left(\mathrm{t}, J=7.2 \mathrm{~Hz}, 1 \mathrm{H}, \mathrm{H}_{\mathrm{AR}}\right), 8.07\left(\mathrm{dd}, J=7.6 \mathrm{~Hz}, 1 \mathrm{H}, \mathrm{H}_{\mathrm{AR}}\right), 12.34$ $(\mathrm{s}, 1 \mathrm{H}, \mathrm{OH}) ;{ }^{13} \mathrm{C}-\mathrm{NMR}\left(100.6 \mathrm{MHz}, \mathrm{CDCl}_{3}\right): \delta=13.8\left(\mathrm{CH}_{3}\right), 23.0(\mathrm{C}-4), 29.6(\mathrm{C}-4 \mathrm{a}), 30.5(\mathrm{C}-8), 35.3(\mathrm{C}-7)$, $35.8\left(\mathrm{NCH}_{3}\right), 46.3(\mathrm{C}-3), 51.8\left(\mathrm{CH}_{3} \mathrm{O}\right), 75.5(\mathrm{C}-8 \mathrm{a}), 96.6(\mathrm{C}-5), 128.3\left(2 \mathrm{CH}_{\mathrm{AR}}\right), 131.0\left(2 \mathrm{CH}_{\mathrm{AR}}\right), 133.9$ $\left(\mathrm{CH}_{\mathrm{AR}}\right), 138.0,\left(\mathrm{C}_{\mathrm{AR}}\right), 165.0(\mathrm{CO}), 171.9$ (C-6, CO); HRMS (ESI) calcd for $\left[\mathrm{C}_{19} \mathrm{H}_{23} \mathrm{NO}_{6} \mathrm{~S}+\mathrm{H}^{+}\right]: 394.1319$, found: 394.1323. Compound 12b: ${ }^{1} \mathrm{H}-\mathrm{NMR}\left(400 \mathrm{MHz}, \mathrm{CDCl}_{3}, \mathrm{COSY}, \mathrm{g}-\mathrm{HSQC}\right): \delta=1.02(\mathrm{~d}, J=7.2 \mathrm{~Hz}$, $\left.3 \mathrm{H}, \mathrm{CH}_{3}\right), 1.69(\mathrm{qd}, J=12.8,4.4 \mathrm{~Hz}, 1 \mathrm{H}, \mathrm{H}-4), 2.16(\mathrm{dd}, J=18.0,1.6 \mathrm{~Hz}, 1 \mathrm{H}, \mathrm{H}-7), 2.32(\mathrm{dm}, J=12.8 \mathrm{~Hz}$, 1H, H-4), $2.77\left(\mathrm{~s}, 3 \mathrm{H}, \mathrm{CH}_{3} \mathrm{~N}\right), 3.04(\mathrm{t}, J=6.8 \mathrm{~Hz}, 1 \mathrm{H}, \mathrm{H}-8), 3.04-3.10(\mathrm{~m}, 2 \mathrm{H}, \mathrm{H}-7, \mathrm{H}-3), 3.25(\mathrm{td}, J=13.2$, $4.0 \mathrm{~Hz}, 1 \mathrm{H}, \mathrm{H}-3), 3.60$ (dd, $J=12.8,4.0 \mathrm{~Hz}, 1 \mathrm{H}, \mathrm{H}-4 \mathrm{a}), 3.81\left(\mathrm{~s}, 3 \mathrm{H}, \mathrm{CH}_{3} \mathrm{O}\right), 7.51\left(\mathrm{t}, J=7.6 \mathrm{~Hz}, 2 \mathrm{H}, \mathrm{H}_{\mathrm{AR}}\right)$, $7.62\left(\mathrm{t}, J=7.2 \mathrm{~Hz}, 1 \mathrm{H}, \mathrm{H}_{\mathrm{AR}}\right), 7.90\left(\mathrm{t}, J=7.2 \mathrm{~Hz}, 1 \mathrm{H}, \mathrm{H}_{\mathrm{AR}}\right), 12.2(\mathrm{~s}, 1 \mathrm{H}, \mathrm{OH}) ;{ }^{13} \mathrm{C}-\mathrm{NMR}(100.6 \mathrm{MHz}$, $\left.\mathrm{CDCl}_{3}\right): \delta=20.6\left(\mathrm{CH}_{3}\right), 28.1(\mathrm{C}-4), 29.7(\mathrm{C}-8), 33.2(\mathrm{C}-7), 33.9(\mathrm{C}-4 \mathrm{a}), 35.8\left(\mathrm{CH}_{3} \mathrm{~N}\right), 48.8(\mathrm{C}-3), 51.8$ $\left(\mathrm{CH}_{3} \mathrm{O}\right), 75.8(\mathrm{C}-8 \mathrm{a}), 97.4(\mathrm{C}-5), 128.3\left(\mathrm{CH}_{\mathrm{AR}}\right), 130.8\left(\mathrm{CH}_{\mathrm{AR}}\right), 133.9(\mathrm{C}-3), 136.7,\left(\mathrm{C}_{\mathrm{AR}}\right), 164.9(\mathrm{CO}), 171.1$ (C-6), $171.9(\mathrm{CO})$.

cis-2-(tert-Butoxycarbonyl)-6-hydroxy-5-(methoxycarbonyl)-1-oxo-8a-(phenylsulfonyl)-1,2,3,4,4a,7,8,8aoctahydroisoquinoline (18a). Operating as described in the general procedure, from unsaturated lactam 6a $(150 \mathrm{mg}, 0.44 \mathrm{mmol})$ in anhydrous $\mathrm{CH}_{2} \mathrm{Cl}_{2}(65 \mathrm{~mL})$, Nazarov reagent 17 (268 $\left.\mathrm{mg}, 1.33 \mathrm{mmol}\right)$, and $\mathrm{Cs}_{2} \mathrm{CO}_{3}(865 \mathrm{mg}, 2.66 \mathrm{mmol})$ in anhydrous $\mathrm{CH}_{2} \mathrm{Cl}_{2}(20 \mathrm{~mL})$, cis-hydroisoquinolone 18a was 
obtained (96 mg, 47\% yield): IR (ATR Pike) $v\left(\mathrm{~cm}^{-1}\right): 1733(\mathrm{C}=\mathrm{O}), 1653(\mathrm{NCO}) ;{ }^{1} \mathrm{H}-\mathrm{NMR}(400 \mathrm{MHz}$, $\left.\left.\mathrm{CDCl}_{3}, \mathrm{COSY}, g-\mathrm{HSQC}\right): \delta=1.55\left[\mathrm{~s}, 9 \mathrm{H},\left(\mathrm{CH}_{3}\right)_{3} \mathrm{C}\right)\right], 1.64(\mathrm{~m}, 1 \mathrm{H}, \mathrm{H}-4), 2.12-2.21(\mathrm{~m}, 3 \mathrm{H}, 2 \mathrm{H}-7, \mathrm{H}-8)$, 2.46-2.54 (m, 2H, H-8, H-4), 3.62-3.74 (m, 2H, 2H-3), $3.85\left(\mathrm{~s}, 3 \mathrm{H}, \mathrm{CH}_{3} \mathrm{O}\right), 3.96(\mathrm{dd}, J=9.6,3.6 \mathrm{~Hz}$, $\mathrm{H}-4 \mathrm{a}), 7.53\left(\mathrm{t}, J=7.6 \mathrm{~Hz}, 2 \mathrm{H}, \mathrm{H}_{\mathrm{AR}}\right), 7.68\left(\mathrm{t}, J=7.6,1.2 \mathrm{~Hz}, 1 \mathrm{H}, \mathrm{H}_{\mathrm{AR}}\right), 7.86(\mathrm{~d}, J=7.6,1.2 \mathrm{~Hz}, 2 \mathrm{H}$, $\left.\mathrm{H}_{\mathrm{AR}}\right), 12.34(\mathrm{~s}, 1 \mathrm{H}, \mathrm{OH}) ;{ }^{13} \mathrm{C}-\mathrm{NMR}\left(100.6 \mathrm{MHz}, \mathrm{CDCl}_{3}\right): \delta=25.8(\mathrm{C}-8), 26.4(\mathrm{C}-7), 27.9\left[\left(\mathrm{CH}_{3}\right)_{3} \mathrm{C}\right], 28.3$ (C-4), $32.6(\mathrm{C}-4 \mathrm{a}), 44.3(\mathrm{C}-3), 51.9\left(\mathrm{CH}_{3} \mathrm{O}\right), 74.1(\mathrm{C}-8 \mathrm{a}), 83.6\left[\left(\mathrm{CH}_{3}\right)_{3} \mathrm{C}\right], 98.4(\mathrm{C}-5), 128.5\left(2 \mathrm{CH}_{\mathrm{AR}}\right), 130.8$ $\left(2 \mathrm{CH}_{\mathrm{AR}}\right), 134.3\left(\mathrm{CH}_{\mathrm{AR}}\right), 135.0\left(\mathrm{C}_{\mathrm{AR}}\right), 151.8(\mathrm{CO}), 166.7(\mathrm{CO}), 171.5,173.9$ (CO, C-6); HRMS (ESI) calcd for $\left[\mathrm{C}_{22} \mathrm{H}_{27} \mathrm{NO}_{8} \mathrm{~S}+\mathrm{NH}_{4}{ }^{+}\right]$: 483.1796 , found: 483.1789 .

cis-6-Hydroxy-5-(methoxycarbonyl)-2-methyl-1-oxo-8a-(phenylsulfonyl)-1,2,3,4,4a,7,8,8a-octahydroisoquinoline (18b). Operating as described in the general procedure, from unsaturated lactam $6 \mathbf{b}(60 \mathrm{mg}$, $0.24 \mathrm{mmol})$ in anhydrous $\mathrm{CH}_{2} \mathrm{Cl}_{2}(35 \mathrm{~mL})$, Nazarov reagent $17(144 \mathrm{mg}, 0.71 \mathrm{mmol})$, and $\mathrm{Cs}_{2} \mathrm{CO}_{3}$ (469 $\mathrm{mg}, 1.44 \mathrm{mmol}$ ) in anhydrous $\mathrm{CH}_{2} \mathrm{Cl}_{2}(15 \mathrm{~mL})$, cis-hydroisoquinolone $\mathbf{1 8 b}$ was obtained (32 $\mathrm{mg}$, $35 \%$ yield): IR (ATR Pike) $v\left(\mathrm{~cm}^{-1}\right): 1653$ (CO); ${ }^{1} \mathrm{H}-\mathrm{NMR}\left(400 \mathrm{MHz}, \mathrm{CDCl}_{3}, \mathrm{COSY}, \mathrm{g}-\mathrm{HSQC}\right): \delta=1.97$ (m, 1H, H-4), 2.00-2.15 (m, 3H, H-7, 2H-8), 2.35 (m, 1H, H-7), 2.46-2.54 (ddt, J = 13.8, 8.8, 4.2 Hz, 1H, $\mathrm{H}-4), 2.93\left(\mathrm{~s}, 3 \mathrm{H}, \mathrm{CH}_{3} \mathrm{~N}\right), 3.17(\mathrm{ddt}, J=12.8,9.4,3.8 \mathrm{~Hz}, 1 \mathrm{H}, \mathrm{H}-3), 3.27(\mathrm{~m}, 1 \mathrm{H}, \mathrm{H}-3), 3.84\left(\mathrm{~s}, 3 \mathrm{H}, \mathrm{CH}_{3} \mathrm{O}\right)$, $4.01(\mathrm{dd}, J=6.4,3.6 \mathrm{~Hz}, \mathrm{H}-4 \mathrm{a}), 7.51\left(\mathrm{t}, J=7.6, \mathrm{~Hz}, 2 \mathrm{H}, \mathrm{H}_{\mathrm{AR}}\right), 7.64\left(\mathrm{t}, J=7.6 \mathrm{~Hz}, 1 \mathrm{H}, \mathrm{H}_{\mathrm{AR}}\right), 7.88(\mathrm{~d}, J=7.6$ $\left.\mathrm{Hz}, 2 \mathrm{H}, \mathrm{H}_{\mathrm{AR}}\right), 12.40(\mathrm{~s}, 1 \mathrm{H}, \mathrm{OH}) ;{ }^{13} \mathrm{C}-\mathrm{NMR}\left(100.6 \mathrm{MHz}, \mathrm{CDCl}_{3}\right): \delta=26.1$ (C-7), $26.2(\mathrm{C}-4), 27.2(\mathrm{C}-8)$, $32.1(\mathrm{C}-4 \mathrm{a}), 35.8\left(\mathrm{CH}_{3} \mathrm{~N}\right), 46.9(\mathrm{C}-3), 51.8\left(\mathrm{CH}_{3} \mathrm{O}\right), 71.6(\mathrm{C}-8 \mathrm{a}), 97.7(\mathrm{C}-5), 128.6\left(2 \mathrm{CH}_{\mathrm{AR}}\right), 130.1\left(2 \mathrm{CH}_{\mathrm{AR}}\right)$, $133.8\left(\mathrm{CH}_{\mathrm{AR}}\right), 136.7\left(\mathrm{C}_{\mathrm{AR}}\right), 164.5(\mathrm{CO}), 171.8,172.9(\mathrm{CO}, \mathrm{C}-6)$; HRMS calcd for $\left[\mathrm{C}_{18} \mathrm{H}_{21} \mathrm{NO}_{6} \mathrm{~S}+\mathrm{H}^{+}\right]$: 380.1162, found: 380.1158 .

cis-6-Hydroxy-5-(methoxycarbonyl)-1-oxo-8a-(phenylsulfonyl)-2-(p-toluenesulfonyl)-1,2,3,4,4a,7,8,8a-octahydroisoquinoline (18c). Operating as described in the general procedure, from unsaturated lactam 6c (50 mg, $0.12 \mathrm{mmol})$ in anhydrous $\mathrm{CH}_{2} \mathrm{Cl}_{2}(20 \mathrm{~mL})$, Nazarov reagent 17 (73 mg, $\left.0.36 \mathrm{mmol}\right)$ and $\mathrm{Cs}_{2} \mathrm{CO}_{3}(234 \mathrm{mg}, 0.72 \mathrm{mmol})$ in anhydrous $\mathrm{CH}_{2} \mathrm{Cl}_{2}(5 \mathrm{~mL})$, cis-hydroisoquinolone $18 \mathrm{c}$ was obtained (20 mg, 32\% overall yield for the three steps). IR (ATR Pike) $v\left(\mathrm{~cm}^{-1}\right): 1653(\mathrm{CO}) ;{ }^{1} \mathrm{H}-\mathrm{NMR}(400 \mathrm{MHz}$, $\mathrm{CDCl}_{3}$, COSY, g-HSQC): $\delta=1.85-1.93(\mathrm{~m}, 2 \mathrm{H}, \mathrm{H}-4, \mathrm{H}-7), 1.99-2.04(\mathrm{~m}, 2 \mathrm{H}, \mathrm{H}-7, \mathrm{H}-8), 2.29-2.35(\mathrm{~m}, 1 \mathrm{H}$, H-8), $2.50\left(\mathrm{~s}, 3 \mathrm{H}, \mathrm{CH}_{3}\right), 2.75-2.70(\mathrm{~m}, 1 \mathrm{H}, \mathrm{H}-4), 3.84\left(\mathrm{~s}, 3 \mathrm{H}, \mathrm{CH}_{3} \mathrm{O}\right) 3.91(\mathrm{~m}, 1 \mathrm{H}, \mathrm{H}-3), 4.01(\mathrm{~m}, 1 \mathrm{H}, \mathrm{H}-3)$, $7.22-7.34\left(\mathrm{~m}, 6 \mathrm{H}, \mathrm{H}_{\mathrm{AR}}\right), 7.60\left(\mathrm{td}, J=7.2,1.2 \mathrm{~Hz}, 1 \mathrm{H}, \mathrm{H}_{\mathrm{AR}}\right), 7.91\left(\mathrm{~d}, J=8.8 \mathrm{~Hz}, 2 \mathrm{H}, \mathrm{H}_{\mathrm{AR}}\right), 12.34(\mathrm{~s}, 1 \mathrm{H}$, $\mathrm{OH}) ;{ }^{13} \mathrm{C}-\mathrm{NMR}\left(100.6 \mathrm{MHz}, \mathrm{CDCl}_{3}\right): \delta=21.7\left(\mathrm{CH}_{3}\right), 25.7$ (C-8), 27.0 (C-7), 28.3 (C-4), 31.9 (C-4a), 43.8 (C-3), 52.0 ( $\left.\mathrm{CH}_{3} \mathrm{O}\right), 73.1(\mathrm{C}-8 \mathrm{a}), 97.8(\mathrm{C}-5), 128.3-130.7\left(\mathrm{CH}_{\mathrm{AR}}\right), 134.3\left(\mathrm{CH}_{\mathrm{AR}}\right), 134.6,135.2\left(\mathrm{C}_{\mathrm{AR}}, \mathrm{CH}_{\mathrm{AR}}\right)$, $145.1\left(\mathrm{C}_{\mathrm{AR}}\right), 165.5(\mathrm{CO}), 171.4,172.9(\mathrm{CO}, \mathrm{C}-6)$; HRMS (ESI) calcd for $\left[\mathrm{C}_{24} \mathrm{H}_{25} \mathrm{NO}_{8} \mathrm{~S}_{2}+\mathrm{H}^{+}\right]$: 520.1094, found: 520.1108 .

cis-2-(tert-Butoxycarbonyl)-8a-(ethoxycarbonyl)-6-hydroxy-5-(methoxycarbonyl)-1-oxo-1,2,3,4,4a,7,8,8aoctahydroisoquinoline (19). Operating as described in the general procedure, from unsaturated lactam 8 (90 mg, $0.33 \mathrm{mmol})$ in anhydrous $\mathrm{CH}_{2} \mathrm{Cl}_{2}(60 \mathrm{~mL})$, Nazarov reagent $17(202 \mathrm{mg}, 1 \mathrm{mmol})$, and $\mathrm{Cs}_{2} \mathrm{CO}_{3}$ (652 mg, $2 \mathrm{mmol}$ ) in anhydrous $\mathrm{CH}_{2} \mathrm{Cl}_{2}(5 \mathrm{~mL})$, cis-hydroisoquinolone 19 was obtained $(87 \mathrm{mg}, 60 \%$ yield): IR (ATR Pike) $v\left(\mathrm{~cm}^{-1}\right)$ : 1725 (CO), $1654(\mathrm{CO}) ;{ }^{1} \mathrm{H}-\mathrm{NMR}\left(400 \mathrm{MHz}, \mathrm{CDCl}_{3}, \mathrm{COSY}, \mathrm{g}-\mathrm{HSQC}\right.$ ): $\left.\delta=1.09\left(\mathrm{~d}, J=7.2 \mathrm{~Hz}, 3 \mathrm{H}, \mathrm{CH}_{2} \mathrm{CH}_{3}\right), 1.36\left[\mathrm{~s}, 9 \mathrm{H}, \mathrm{CH}_{3}\right)_{3} \mathrm{C}\right], 1.51(\mathrm{ddd}, J=14.0,12.0,4.8 \mathrm{~Hz}, 1 \mathrm{H}, \mathrm{H}-4)$, 1.90 (ddd, $J=14.0,11.2,7.2 \mathrm{~Hz}, 1 \mathrm{H}, \mathrm{H}-8), 2.03(\mathrm{dq}, J=14.0,3.6 \mathrm{~Hz}, 1 \mathrm{H}, \mathrm{H}-4), 2.18$ (ddd, $J=18.4,7.2,2.0$ $\mathrm{Hz}, 1 \mathrm{H}, \mathrm{H}-7), 2.32$ (dd, $J=14.0,7.2 \mathrm{~Hz}, 1 \mathrm{H}, \mathrm{H}-8), 2.63(\mathrm{ddd}, J=18.4,10.4,8.0 \mathrm{~Hz}, 1 \mathrm{H}, \mathrm{H}-7), 3.31$ (dd, $J=11.6,3.2 \mathrm{~Hz}, 1 \mathrm{H}, \mathrm{H}-4 \mathrm{a}), 3.40(\mathrm{td}, J=12.4,3.6 \mathrm{~Hz}, 1 \mathrm{H}, \mathrm{H}-3), 3.64\left(\mathrm{~s}, 3 \mathrm{H}, \mathrm{CH}_{3} \mathrm{O}\right), 3.66(\mathrm{ddd}, J=12.4$, 4.8, 3.2 Hz, $1 \mathrm{H}, \mathrm{H}-3), 4.05$ (qd, $\left.J=7.2,2.4 \mathrm{~Hz}, 2 \mathrm{H}, \mathrm{CH}_{2} \mathrm{CH}_{3}\right), 12.18(\mathrm{~s}, 1 \mathrm{H}, \mathrm{OH}) ;{ }^{13} \mathrm{C}-\mathrm{NMR}(100.6 \mathrm{MHz}$, $\left.\mathrm{CDCl}_{3}\right): \delta=13.9\left(\mathrm{CH}_{2} \mathrm{CH}_{3}\right), 24.6(\mathrm{C}-8), 26.3(\mathrm{C}-7), 27.5(\mathrm{C}-4), 27.9\left[\left(\mathrm{CH}_{3}\right)_{3} \mathrm{C}\right], 34.7(\mathrm{C}-4 \mathrm{a}), 45.7(\mathrm{C}-3), 51.7$ $\left(\mathrm{CH}_{3} \mathrm{O}\right), 56.1(\mathrm{C}-8 \mathrm{a}), 61.7\left(\mathrm{CH}_{2} \mathrm{CH}_{3}\right), 83.4\left[\left(\mathrm{CH}_{3}\right)_{3} \mathrm{C}\right], 98.0(\mathrm{C}-5), 152.7(\mathrm{C}-6), 170.4(\mathrm{CO}), 170.5(\mathrm{CO})$, 171.9 (CO), 172.7 (CO); HRMS (ESI) calcd for $\left[\mathrm{C}_{19} \mathrm{H}_{27} \mathrm{NO}_{8}+\mathrm{Na}^{+}\right]: 420.1629$, found: 420.1645.

\subsection{General Procedure for the Desulfonylation Reactions}

$\mathrm{Na}_{2} \mathrm{HPO}_{4}$ (50 equiv.) and sodium amalgam (25 equiv.) were added at $-78{ }^{\circ} \mathrm{C}$ under an argon atmosphere to a solution of the sulfonyl derivative $10 \mathbf{a}, \mathbf{1 2} \mathbf{a}$ or $\mathbf{1 2 b}$ (1 equiv.) in anhydrous methanol 
$(0.025 \mathrm{M})$, and the mixture was stirred at $-78^{\circ} \mathrm{C}$ for $2 \mathrm{~h}$. The solution was then filtered and quenched with $\mathrm{H}_{2} \mathrm{O}$ at low temperature. The mixture was concentrated under reduced pressure, and the resulting aqueous solution was extracted with EtOAc. The combined organic extracts were dried over anhydrous $\mathrm{MgSO}_{4}$, filtered, and concentrated under reduced pressure. Flash chromatography (9:1 hexane-EtOAc) of the residue afforded the desulfurated compounds 13a, 14a or 14b (detailed data in supplementary materials).

cis-2-(tert-Butoxycarbonyl)-6-hydroxy-5-(methoxycarbonyl)-8-methyl-1-oxo-1,2,3,4,4a,7,8,8a-octahydroisoquinoline ( $\mathrm{H}-8 / \mathrm{H}-8$ a trans, 13a). Operating as described in the above general desulfonylation procedure, from a solution of sulfonyl derivative $\mathbf{1 0 a}(580 \mathrm{mg}, 1.21 \mathrm{mmol})$ in anhydrous methanol $(50 \mathrm{~mL})$, $\mathrm{Na}_{2} \mathrm{HPO}_{4}(8.60 \mathrm{~g}, 60.6 \mathrm{mmol})$, and sodium amalgam $(6.77 \mathrm{~g}, 30.3 \mathrm{mmol})$, compound 13a was obtained (357 mg, 87\% yield): ${ }^{1} \mathrm{H}-\mathrm{NMR}\left(400 \mathrm{MHz}, \mathrm{CDCl}_{3}, \mathrm{COSY}, \mathrm{g}-\mathrm{HSQC}\right): \delta=1.05\left(\mathrm{~d}, \mathrm{~J}=6.0 \mathrm{~Hz}, 3 \mathrm{H}, \mathrm{CH}_{3}\right)$, 1.53 [s, 9H, $\left.\left(\mathrm{CH}_{3}\right)_{3} \mathrm{C}\right], 1.79$ (dddd, $\left.J=19.2,12.8,11.2,4.8 \mathrm{~Hz}, 1 \mathrm{H}, \mathrm{H}-4\right), 2.04$ (m, 1H, H-7), 2.10 (m, 1H, H-4), 2.30 (m, 2H, H-8, H-8a), 2,46 (dd, $J=19.2,4.8 \mathrm{~Hz}, 1 \mathrm{H}, \mathrm{H}-7), 2.98$ (dt, J = 11.2, 4.0 Hz, 1H, $\mathrm{H}-4 \mathrm{a}), 3.41$ (td, $J=12.8,4.0 \mathrm{~Hz}, 1 \mathrm{H}, \mathrm{H}-3), 3.70-3.75$ (m, 1H, H-3), 3.79 (s, 3H, $\left.\mathrm{CH}_{3} \mathrm{O}\right), 12.23(\mathrm{~s}, 1 \mathrm{H}, \mathrm{OH})$; ${ }^{13} \mathrm{C}-\mathrm{NMR}\left(100.6 \mathrm{MHz}, \mathrm{CDCl}_{3}\right): \delta=18.8\left(\mathrm{CH}_{3}\right), 26.9(\mathrm{C}-8), 27.1(\mathrm{C}-4), 27.9\left[\left(\mathrm{CH}_{3}\right)_{3} \mathrm{C}\right], 32.0(\mathrm{C}-4 \mathrm{a}), 36.8$ (C-7), 45.2 (C-3), $50.5(\mathrm{C}-8 \mathrm{a}), 51.6\left(\mathrm{CH}_{3} \mathrm{O}\right), 82.7$ [ $\left.\left(\mathrm{CH}_{3}\right)_{3} \mathrm{C}\right], 99.3(\mathrm{C}-5), 153.3(\mathrm{CO}), 169.0(\mathrm{CO}), 172.2$, 173.1 (CO, C-6); HRMS (ESI) calcd for $\left[\mathrm{C}_{17} \mathrm{H}_{25} \mathrm{NO}_{6}+\mathrm{Na}^{+}\right]$: 362.1574, found: 362.1579 .

cis-6-Hydroxy-5-(methoxycarbonyl)-2-methyl-1-oxo-1,2,3,4,4a,7,8,8a-octahydroisoquinoline (H-8/H-8a trans, 14a). Operating as described in the general desulfonylation procedure, from a solution of sulfone 12a (100 mg, $0.25 \mathrm{mmol})$ in anhydrous $\mathrm{MeOH}(10 \mathrm{~mL}), \mathrm{Na}_{2} \mathrm{HPO}_{4}(1.81 \mathrm{~g}, 12.7 \mathrm{mmol})$, and sodium amalgam (1.42 g, $6.36 \mathrm{mmol})$, compound 14a was obtained (48 $\mathrm{mg}, 76 \%$ yield) as a white foam: IR (ATR Pike) $v\left(\mathrm{~cm}^{-1}\right): 1645(\mathrm{CO}) ;{ }^{1} \mathrm{H}-\mathrm{NMR}\left(400 \mathrm{MHz}, \mathrm{CDCl}_{3}, \mathrm{COSY}, \mathrm{g}-\mathrm{HSQC}\right): \delta=1.09(\mathrm{~d}, \mathrm{~J}=7.2 \mathrm{~Hz}$, $3 \mathrm{H}, \mathrm{CH}_{3}$ ), 1.90 (m, 1H, H-4), 1.94-2.10 (m, 2H, H-4, H-7), 2.24 (m, 2H, H-8, H-8a), 2.38 (d, J = 18.4, $4.4 \mathrm{~Hz}, 1 \mathrm{H}, \mathrm{H}-7), 2.90$ (m, 1H, H-4a), $2.92\left(\mathrm{~s}, 3 \mathrm{H}, \mathrm{CH}_{3} \mathrm{~N}\right), 3.21-3.25(\mathrm{~m}, 2 \mathrm{H}, \mathrm{H}-3), 3.79\left(\mathrm{~s}, 3 \mathrm{H}, \mathrm{CH}_{3} \mathrm{O}\right)$, $12.30(\mathrm{~s}, 1 \mathrm{H}, \mathrm{OH}) ;{ }^{13} \mathrm{C}-\mathrm{NMR}\left(100.6 \mathrm{MHz}^{\mathrm{C}} \mathrm{CDCl}_{3}\right): \delta=19.2\left(\mathrm{CH}_{3}\right), 26.5(\mathrm{C}-4), 27.4(\mathrm{C}-8 \mathrm{a}), 31.3(\mathrm{C}-4 \mathrm{a})$, $34.8\left(\mathrm{CH}_{3} \mathrm{~N}\right), 37.1(\mathrm{C}-7), 47.2(\mathrm{C}-8), 48.8(\mathrm{C}-3), 51.5\left(\mathrm{CH}_{3} \mathrm{O}\right), 99.1(\mathrm{C}-5), 170.7(\mathrm{CO}), 172.3(\mathrm{C}-6), 172.6$ (CO); HRMS (ESI) calcd for $\left[\mathrm{C}_{13} \mathrm{H}_{20} \mathrm{NO}_{4}+\mathrm{H}^{+}\right]$: 254.1387, found: 254.1387.

cis-6-Hydroxy-5-(methoxycarbonyl)-2-methyl-1-oxo-1,2,3,4,4a,7,8,8a-octahydroisoquinoline (H-8/H-8a cis, 14b). Operating as described in the general desulfonylation procedure, from a solution of sulfone $12 b$ (340 mg, $0.87 \mathrm{mmol})$ in anhydrous $\mathrm{MeOH}(10 \mathrm{~mL}), \mathrm{Na}_{2} \mathrm{HPO}_{4}(6.134 \mathrm{~g}, 43.2 \mathrm{mmol})$, and sodium amalgam $(4.836 \mathrm{~g}, 21.6 \mathrm{mmol})$, compound $\mathbf{1 4 b}$ was obtained $(137 \mathrm{mg}, 62 \%$ yield) as a white foam: ${ }^{1} \mathrm{H}-\mathrm{NMR}\left(400 \mathrm{MHz} \mathrm{CDCl}_{3}, \mathrm{COSY}, \mathrm{g}-\mathrm{HSQC}\right): \delta=1.05\left(\mathrm{~d}, J=7.2 \mathrm{~Hz}, 3 \mathrm{H}, \mathrm{CH}_{3}\right.$ ), $1.86(\mathrm{qd}, J=12.0,5.2$, 1H, H-4), 2.10 (dm, $J=12.0 \mathrm{~Hz}, 1 \mathrm{H}, \mathrm{H}-4), 2.19$ (dd, J = 18.0, $2.0 \mathrm{~Hz}, 1 \mathrm{H}, \mathrm{H}-7), 2.657-2.64$ (m, 12H, H-8, H-7), 2.67 (m, 1H, H-8a), 2.85-2.90 (m, 1H, H-4a), 3.00 (s, 3H, $\left.\mathrm{NCH}_{3}\right), 3.24$ (dq, J = 12.0, 3.2 Hz, 1H, H-3), 3.39 (td, $J=12.0,4.4 \mathrm{~Hz}, 1 \mathrm{H}, \mathrm{H}-3), 3.79$ (s, 3H, $\left.\mathrm{CH}_{3} \mathrm{O}\right), 12.48$ (s, $\left.1 \mathrm{H}, \mathrm{OH}\right) ;{ }^{13} \mathrm{C}-\mathrm{NMR}(100.6 \mathrm{MHz}$, $\left.\mathrm{CDCl}_{3}\right): \delta=18.8\left(\mathrm{CH}_{3}\right), 26.6(\mathrm{C}-4), 29.4(\mathrm{C}-8 \mathrm{a}), 31.9(\mathrm{C}-4 \mathrm{a}), 34.9\left(\mathrm{CH}_{3} \mathrm{~N}\right), 36.1(\mathrm{C}-7), 43.6(\mathrm{C}-8), 49.2(\mathrm{C}-3)$, $51.5\left(\mathrm{CH}_{3} \mathrm{O}\right), 98.0(\mathrm{C}-5), 171.2(\mathrm{CO}), 172.6(\mathrm{C}-6, \mathrm{CO})$.

\subsection{General Procedure for the Removal of the N-Boc Substituent}

TFA (1:3 TFA $/ \mathrm{CH}_{2} \mathrm{Cl}_{2}$ ratio) was added to a solution of the $N$-Boc derivative 10a or 13a in anhydrous $\mathrm{CH}_{2} \mathrm{Cl}_{2}(0.05-0.06 \mathrm{M})$, and the mixture was stirred for 30 minutes at room temperature. Toluene $(1 \mathrm{~mL})$ was added to the resulting solution, and the mixture was concentrated under reduced pressure. After a second addition of toluene $(1 \mathrm{~mL})$ and concentration of the mixture, flash chromatography (7:3 to 3:7 hexane-EtOAc) of the residue afforded the deprotected compound 15 or 16.

cis-6-Hydroxy-5-(methoxycarbonyl)-8-methyl-1-oxo-8a-(phenylsulfonyl)-1,2,3,4,4a,7,8,8a-octahydroisoquinoline $\left(\mathrm{Me} / \mathrm{SO}_{2} \mathrm{Ph}\right.$ cis, 15). Operating as described in the above general procedure, from a solution of the $N$-Boc derivative $10 a(23 \mathrm{mg}, 0.048 \mathrm{mmol})$ in anhydrous $\mathrm{CH}_{2} \mathrm{Cl}_{2}(750 \mu \mathrm{L})$ and TFA $(250 \mu \mathrm{L})$, compound 15 was obtained $\left(18 \mathrm{mg}, 98 \%\right.$ yield): IR (ATR Pike) $v\left(\mathrm{~cm}^{-1}\right): 3352(\mathrm{NH}), 1668(\mathrm{CO}) ;{ }^{1} \mathrm{H}-\mathrm{NMR}(400$ 
$\mathrm{MHz}, \mathrm{CDCl}_{3}, \mathrm{COSY}$, g-HSQC): $\delta=1.11\left(\mathrm{~d}, J=6.8 \mathrm{~Hz}, 3 \mathrm{H}, \mathrm{CH}_{3}\right), 1.94(\mathrm{dd}, J=17.6,2.0 \mathrm{~Hz}, \mathrm{H}-7), 2.32$ $(\mathrm{dm}, J=14.8 \mathrm{~Hz}, 1 \mathrm{H}, \mathrm{H}-4), 2.45$ (m, 1H, H-8), 2.50 (dm, $J=17.6 \mathrm{~Hz}, 1 \mathrm{H}, \mathrm{H}-7), 2.65$ (dddd, $J=14.8,12.4$, 5.6, 3.6 Hz, 1H, H-4), 3.16 (td, $J=12.4,4.4 \mathrm{~Hz}, 1 \mathrm{H}, \mathrm{H}-3), 3.27$ (m, 1H, H-3), 3.85 (s, 3H, $\mathrm{CH}_{3} \mathrm{O}$ ), 3.97 (br.s, 1H, H-4a), 6.29 (br.s, 1H, NH), $7.53\left(\mathrm{t}, J=7.2, \mathrm{~Hz}, 2 \mathrm{H}, \mathrm{H}_{\mathrm{AR}}\right), 7.65\left(\mathrm{t}, J=7.2 \mathrm{~Hz}, 1 \mathrm{H}, \mathrm{H}_{\mathrm{AR}}\right), 8.06$ $\left(\mathrm{d}, J=7.2 \mathrm{~Hz}, 2 \mathrm{H}, \mathrm{H}_{\mathrm{AR}}\right), 12.33(\mathrm{~s}, 1 \mathrm{H}, \mathrm{OH}) ;{ }^{13} \mathrm{C}-\mathrm{NMR}\left(100.6 \mathrm{MHz}, \mathrm{CDCl}_{3}\right): \delta=13.7\left(\mathrm{CH}_{3}\right), 22.4(\mathrm{C}-4)$, 29.2 (C-4a), 30.1 (C-8), 35.2 (C-7), 38.7 (C-3), $51.8\left(\mathrm{CH}_{3} \mathrm{O}\right), 75.1$ (C-8a), $96.5(\mathrm{C}-5), 128.3\left(2 \mathrm{CH}_{\mathrm{AR}}\right), 130.6$ $\left(2 \mathrm{CH}_{\mathrm{AR}}\right), 134.0\left(\mathrm{CH}_{\mathrm{AR}}\right), 137.7\left(\mathrm{C}_{\mathrm{AR}}\right), 167.0(\mathrm{CO}), 171.6,171.8(\mathrm{CO}, \mathrm{C}-6)$; HRMS calcd for $\left[\mathrm{C}_{18} \mathrm{H}_{21} \mathrm{NO}_{6} \mathrm{~S}\right.$ $+\mathrm{H}^{+}$]: 380.1162 , found: 380.1164 .

cis-6-Hydroxy-5-(methoxycarbonyl)-8-methyl-1-oxo-1,2,3,4,4a,7,8,8a-octahydroisoquinoline (H-8/H-8a trans, 16). Operating as described in the above general procedure, from a solution of the $\mathrm{N}$-Boc derivative 13a $(10 \mathrm{mg}, 0.03 \mathrm{mmol})$ in anhydrous $\mathrm{CH}_{2} \mathrm{Cl}_{2}(600 \mu \mathrm{L})$ and TFA $(200 \mu \mathrm{L})$, compound 16 was obtained (7 mg, 99\% yield): IR (ATR Pike) $v\left(\mathrm{~cm}^{-1}\right): 3340(\mathrm{NH}), 1659(\mathrm{CO}) ;{ }^{1} \mathrm{H}-\mathrm{NMR}\left(400 \mathrm{MHz}, \mathrm{CDCl}_{3}, \mathrm{COSY}\right.$, g-HSQC): $\delta=1.12\left(\mathrm{~d}, J=6.8 \mathrm{~Hz}, 3 \mathrm{H}, \mathrm{CH}_{3}\right), 1.83(\mathrm{~m}, 1 \mathrm{H}, \mathrm{H}-4), 1.98(\mathrm{dd}, J=13.6,3.6 \mathrm{~Hz}, 1 \mathrm{H}, \mathrm{H}-4), 2.06$ (dd, $J=18.4,8.8 \mathrm{~Hz}, \mathrm{H}-7), 2.23(\mathrm{~m}, 2 \mathrm{H}, \mathrm{H}-8, \mathrm{H}-8 \mathrm{a}), 2.42(\mathrm{dd}, J=18.4,4.8 \mathrm{~Hz}, 1 \mathrm{H}, \mathrm{H}-7), 2.93(\mathrm{~m}, 1 \mathrm{H}$, $\mathrm{H}-4 \mathrm{a}), 3.23-3.40$ (m, 2H, H-3), 3.79 (s, 3H, $\left.\mathrm{CH}_{3} \mathrm{O}\right), 6.22$ (br.s, $\left.1 \mathrm{H}, \mathrm{NH}\right), 12.29(\mathrm{~s}, 1 \mathrm{H}, \mathrm{OH})$; ${ }^{13} \mathrm{C}-\mathrm{NMR}(100.6$ $\left.\mathrm{MHz}, \mathrm{CDCl}_{3}\right): \delta=19.1\left(\mathrm{CH}_{3}\right), 25.7(\mathrm{C}-4), 27.3(\mathrm{C}-8, \mathrm{C}-8 \mathrm{a}), 31.0(\mathrm{C}-4 \mathrm{a}), 37.1(\mathrm{C}-7), 41.1(\mathrm{C}-3), 51.6\left(\mathrm{CH}_{3} \mathrm{O}\right)$, 99.1 (C-5), 172.2, 172.4 (CO, C-6); HRMS calcd for $\left[\mathrm{C}_{12} \mathrm{H}_{17} \mathrm{NO}_{4}+\mathrm{H}^{+}\right]$: 240.1230, found: 240.1232.

Supplementary Materials: The following are available online. Copies of ${ }^{1} \mathrm{H},{ }^{13} \mathrm{C}$, and NOE NMR spectra, and crystallographic data for 10a (CCDC 1890851), 12a (CCDC 1890850), and 12b (CCDC 1890852). CCDC 1890850-1890852 contains the supplementary crystallographic data for this paper. These data can be obtained free of charge via http:/ / www.ccdc.cam.ac.uk/conts/retrieving.html (or from the CCDC, 12 Union Road, Cambridge CB2 1EZ, UK; Fax: +44 1223 336033; E-mail: deposit@ccdc.cam.ac.uk).

Author Contributions: M.A. designed and planned the research; M.P. supervised the experimental work; F.A. and C.A. performed the experimental work and characterized the compounds; E.M. carried out the X-ray analysis; J.B. discussed the results and prepared the manuscript for publication.

Funding: This research was funded by the MINECO/FEDER, Spain (Project CTQ2015-65384-R).

Acknowledgments: Financial support from the MINECO/FEDER, Spain (Project CTQ2015-65384-R) is gratefully acknowledged. Thanks are also due to the MINECO (Spain) for a fellowship to F. A. (AP2010-1663). We also acknowledge the networking contribution from the COST Action CM1407.

Conflicts of Interest: The authors declare no conflict of interest.

\section{References}

1. Taylor, D.R.; MacCoss, M.; Lawson, A.D.G. Rings in drugs. J. Med. Chem. 2014, 57, 5845-5859. [CrossRef] [PubMed]

2. Vitaku, E.; Smith, D.T.; Njardarson, J.T. Analysis of the structural diversity, substitution patterns, and frequency of nitrogen heterocycles among U.S. FDA approved pharmaceuticals. J. Med. Chem. 2014, 57, 10257-10274. [CrossRef] [PubMed]

3. Brown, R.T. The yohimbine group. Monoterpenoid Indole Alkaloids. In The Chemistry of Heterocyclic Compounds; Saxton, J.E., Ed.; John Wiley and Sons: New York, NY, USA, 1983; Volume 25, pp. 147-200.

4. Szántay, C.; Honty, K. The yohimbine group. Monoterpenoid Indole Alkaloids. In The Chemistry of Heterocyclic Compounds; Saxton, J.E., Ed.; John Wiley and Sons: New York, NY, USA, 1994; Volume 25, pp. 161-216.

5. Peng, J.; Rao, K.V.; Choo, Y.-M.; Hamann, M.T. Manzamine alkaloids. In Modern Alkaloids: Structure, Isolation, Synthesis and Biology; Fattorusso, E., Taglialatela-Scafati, O., Eds.; Wiley-VCH: Weinheim, Germany, 2008; pp. 189-232.

6. Amat, M.; Perez, M.; Ballette, R.; Proto, S.; Bosch, J. The alkaloids of the madangamine group. In The Alkaloids; Knölker, H.-J., Ed.; Elsevier: Oxford, UK, 2015; Volume 74, pp. 159-199.

7. Kaldor, S.W.; Kalish, V.J.; Davies, J.F., II; Shetty, B.V.; Fritz, J.E.; Appelt, K.; Burgess, J.A.; Campanale, K.M.; Chirgadze, N.Y.; Clawson, D.K.; et al. Viracept (nelfinavir mesylate, AG1343): A potent, orally bioavailable inhibitor of HIV-1 protease. J. Med. Chem. 1997, 40, 3979-3985. [CrossRef] [PubMed] 
8. Arusksakunwong, O.; Promsri, S.; Witta, K.; Nimmanpipug, P.; Lee, V.S.; Wijitkosoom, A.; Sompornpisut, P.; Hannongbua, S. Current development on HIV-1 protease inhibitors. Curr. Comput. Aided Drug Des. 2007, 3, 201-213. [CrossRef]

9. Weiss, B.; Alt, A.; Ogden, A.M.; Gates, M.; Dieckman, D.K.; Clemens-Smith, A.; Ho, K.H.; Jarvie, K.; Rizkalla, G.; Wright, R.A.; et al. Pharmacological characterization of the competitive GLUK5 receptor antagonist decahydroisoquinoline LY466195 in vitro and in vivo. Pharmacol. Exp. Ther. 2006, 318, 772-781. [CrossRef] [PubMed]

10. Motani, A.S.; Luo, J.; Liang, L.; Mihalic, J.T.; Chen, X.; Tang, L.; Li, L.; Jaen, J.; Chen, J.-L.; Dai, K. Evaluation of AMG 076, a potent and selective MCHR1 antagonist, in rodent and primate obesity models. Pharmacol. Res. Perspect. 2013, 1, e00003. [CrossRef] [PubMed]

11. Martin, S.F.; Williamson, S.A.; Gist, R.P.; Smith, K.M. Aspects of the intramolecular Diels-Alder reactions of some 1,3,9-trienic amides, amines, and esters. An approach to the pentacyclic skeleton of the yohimboid alkaloids. J. Org. Chem. 1983, 48, 5170-5180. [CrossRef]

12. Moriwake, T.; Hamano, S.; Saito, S.; Torii, S.; Kashino, S. Synthesis of the chiral (8S)-7-aza-1,3(E),9-decatriene system from natural. $\alpha$-Amino acids and its intramolecular Diels-Alder reaction directed toward chiral trans-hydroisoquinolones. J. Org. Chem. 1989, 54, 4114-4120. [CrossRef]

13. Casamitjana, N.; López, V.; Jorge, A.; Bosch, J.; Molins, E.; Roig, A. Diels-Alder reactions of 5,6-dihydro-2(1H)pyridones. Tetrahedron 2000, 56, 4027-4042. [CrossRef]

14. Zibuck, R. t-Butyl (E)-3-oxo-4-hexenoate. In Encyclopedia of Reagents for Organic Synthesis; Paquette, L.A., Ed.; Wiley: Chichester, UK, 1995; Volume 2, pp. 936-937.

15. Zibuck, R. Methyl 3-oxo-4-pentenoate. In Encyclopedia of Reagents for Organic Synthesis; Paquette, L.A., Ed.; Wiley: Chichester, UK, 1995; Volume 5, pp. 3558-3559.

16. Audran, G.; Brémond, P.; Feuerstein, M.; Marque, S.R.A.; Santelli, M. Nazarov reagents and their use in organic synthesis. Tetrahedron 2013, 69, 8325-8348. [CrossRef]

17. Amat, M.; Arioli, F.; Pérez, M.; Molins, E.; Bosch, J. Preparation and double Michael addition reactions of a synthetic equivalent of the Nazarov reagent. Org. Lett. 2013, 15, 2470-2473. [CrossRef] [PubMed]

18. Arioli, F.; Pérez, M.; Are, C.; Estarellas, C.; Luque, F.J.; Bosch, J.; Amat, M. Stereocontrolled annulations of indolo[2,3-a]quinolizidine-derived lactams with a silylated Nazarov reagent. Access to allo and epiallo yohimbine-type derivatives. Chem. Eur. J. 2015, 21, 13382-13389. [CrossRef] [PubMed]

19. Estarellas, C.; Arioli, F.; Pérez, M.; Are, C.; Hevia, D.; Molins, E.; Luque, F.J.; Bosch, J.; Amat, M. Origin of the base-dependent facial selectivity in annulation reactions of Nazarov-type reagents with unsaturated indolo[2,3-a]quinolizidine lactams. Eur. J. Org. Chem. 2017, 3969-3979. [CrossRef]

20. Lavallée, J.-F.; Deslongchamps, P. Synthesis of cis-decalin via Diels-Alder and double Michael cycloaddition with substituted Nazarov reagent. Tetrahedron Lett. 1988, 29, 5117-5118. [CrossRef]

21. Lavallée, J.-F.; Spino, C.; Ruel, R.; Hogan, K.T.; Deslongchamps, P. Stereoselective synthesis of cis-decalins via Diels-Alder and double Michael addition of substituted Nazarov reagents. Can. J. Chem. 1992, 70, 1406-1426. [CrossRef]

22. Jung, Y.C.; Yoon, C.H.; Turos, E.; Yoo, K.S.; Jung, K.J. Total Syntheses of (-)- $\alpha$-kainic acid and (+)- $\alpha$-allokainic acid via stereoselective C-H insertion and efficient 3,4-stereocontrol. J. Org. Chem. 2007, 72, 10114-10122. [CrossRef] [PubMed]

Sample Availability: Samples of the compounds are not available from the authors. 